\title{
Dark energy and inflation invoked in CCGG by locally contorted space-time
}

\author{
David Vasak $^{1, \mathrm{a}}{ }_{\mathbb{D}}$, Johannes Kirsch ${ }^{1}$, Jürgen Struckmeier ${ }^{1,2}$ \\ ${ }^{1}$ Frankfurt Institute for Advanced Studies (FIAS), Ruth-Moufang-Strasse 1, 60438 Frankfurt am Main, \\ Germany \\ ${ }^{2}$ Goethe Universität, Max-von-Laue-Strasse 1, 60438 Frankfurt am Main, Germany
}

Received: 4 October 2019 / Accepted: 23 April 2020 / Published online: 19 May 2020

(C) The Author(s) 2020

\begin{abstract}
The cosmological implications of the covariant canonical gauge theory of gravity (CCGG) are investigated. We deduce that, in a metric-compatible geometry, the requirement of covariant conservation of matter invokes torsion of space-time. In the Friedman model, this leads to a scalar field built from contortion and the metric with the property of dark energy, which transforms the cosmological constant to a time-dependent function. Moreover, the quadratic, scale-invariant Riemann-Cartan term in the CCGG Lagrangian endows spacetime with kinetic energy, and in the field equations adds a geometrical curvature correction to Einstein gravity. Applying in the Friedman model the standard $\Lambda \mathrm{CDM}$ parameter set, those equations yield a cosmological field depending just on one additional, dimensionless "deformation" parameter of the theory that determines the strength of the quadratic term, viz. the deviation from the Einstein-Hilbert ansatz. Moreover, the apparent curvature of the universe differs from the actual curvature parameter of the metric. The numerical analysis in that parameter space yields three cosmology types: (1) a bounce universe starting off from a finite scale followed by a steady inflation, (2) a singular Big Bang universe undergoing a secondary inflation-deceleration phase and (3) a solution similar to standard cosmology but with a different temporal profile. The common feature of all scenarios is the graceful exit to the current dark energy era. The value of the deformation parameter can be deduced by comparing theoretical calculations with observations, namely with the SNeIa Hubble diagram and the deceleration parameter. That comparison implies a considerable admixture of scale-invariant quadratic gravity to Einstein gravity. This theory also sheds new light on the resolution of the cosmological constant problem and of the Hubble tension.
\end{abstract}

\section{Introduction}

The nature of dark matter and dark energy is a long-standing unresolved problem in current cosmology. These ingredients have been added to Einstein's general relativity (GR) in order to account for the observed accelerating expansion and the missing mass in the universe. An alternative direction to account for these observations has been to modify the EinsteinHilbert theory by higher-order curvature terms and/or auxiliary scalar fields $[40,41,52,53]$.

\footnotetext{
a e-mail: vasak@ fias.uni-frankfurt.de (corresponding author)
} 
Here, we consider the cosmological impact of the covariant canonical gauge theory of gravity (CCGG) [42-46], a classical Palatini field theory extending Einstein gravity by a quadratic Riemann-Cartan invariant.

The paper is organized as follows. In Sect. 2, we sketch the field-theoretical basis of the gauge theory of gravity, an approach in the spirit of earlier work on gauge theories of gravitation $[23,24,27,39,48]$, but relying on the mathematical rigorousness of the canonical transformation theory in the Hamiltonian picture. That framework leads to a modified version of the Einstein equation in which torsion is admitted and metric compatibility dynamically implemented. In Sect. 3, we discuss why torsion is a necessary degree of freedom in the theory, how the cosmological constant is promoted to a cosmological field and how the quadratic extension in the Hamiltonian gives rise to a geometrical stress tensor. We do not involve any auxiliary dynamical scalar fields nor do we invoke any further ad hoc modifications of Einstein gravity.

The CCGG-Friedman model, in which dynamic dark energy and curvature appear as energy stores ${ }^{1}$, is reviewed in Sect. 4, with details collected in the "Appendix." Not only becomes the cosmological "constant" a time-dependent quantity but also the apparent curvature, while the curvature parameter of the FLRW metric remains unchanged. The numerical solutions are discussed in Sect. 5. By setting the priors to coincide with the $\Lambda$ CDM parameter set, the geometry-induced corrections give rise to a time-dependent dark energy function. The evolution of the universe is then determined by the new, dimensionless parameter of the theory that fixes the strength of the quadratic, scale-invariant term relative to Einstein gravity (thus called the deformation parameter).

The deformation parameter also influences the redshift dependence of the theoretical luminosity-distance modulus, and its comparison with the SNeIa Hubble diagram allows to gain information on its value (cf. Sect. 5.4). Moreover, in Sect. 6, the relation of the free parameter of the CCGG theory with the value of the cosmological constant is discussed.

The paper closes with a Summary and conclusions section.

\section{The covariant canonical gauge theory of gravity}

The application of the canonical Hamiltonian transformation theory to classical relativistic matter fields has been pioneered by Struckmeier et al. and proven to derive the Yang-Mills gauge theory from first principles $[42,45]$. At the heart of this framework is the requirement that the system dynamics is given by an action integral that remains invariant under prescribed local transformations of the original (matter) fields. Those transformations are implemented in the covariant de Donder-Hamiltonian formalism [18] by the choice of a generating function, specifically designed for any given underlying symmetry group. That formalism unambiguously introduces symmetry-dependent gauge fields and fixes their interaction with the matter fields. The kinetic portion of the newly introduced gauge fields, i.e., the Hamiltonian of noninteracting gravity, is not entirely determined by the gauging process, though. It is rather introduced as an educated guess based on physical considerations and empirical insights.

Applying the above framework to the diffeomorphism group paves a novel path to implementing Einstein's principle of general relativity to arbitrary classical relativistic systems of matter fields. In the resulting first-order theory, the space-time geometry is described by both the affine connection $\gamma_{\beta \mu}^{\alpha}$ that is not necessarily symmetric in $\beta$ and $\mu$, and the independent

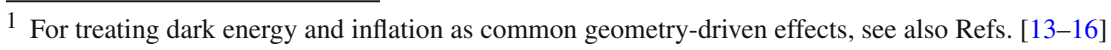


metric tensor $g_{\mu \nu}{ }^{2}$. The fundamental fields describing the dynamics of gravitation encompass, in addition to the connection and metric, also the "affine momentum" fields, the rank-4 tensor density conjugate to the affine connection, and the "metric momentum" field, a rank-3 tensor density conjugate to the metric. The Hamiltonian (scalar) density, $\tilde{\mathcal{H}}_{\mathrm{Gr}}$, of space-time dynamics extends the Einstein-Hilbert ansatz by a quadratic invariant built from the affine momentum tensor $[5,44]$. In order to comply with the key observations that already gave credibility to Einstein's equation [44], we set

$$
\tilde{\mathcal{H}}=\tilde{\mathcal{H}}_{\text {Gr }}+\tilde{\mathcal{H}}_{\text {matter }}
$$

with $^{3}$

$$
\tilde{\mathcal{H}}_{\mathrm{Gr}}=\frac{1}{4 g_{1} \sqrt{-g}} \tilde{q}_{\eta}{ }^{\alpha \xi \beta} \tilde{q}_{\alpha}{ }^{\eta \tau \lambda} g_{\xi \tau} g_{\beta \lambda}-g_{2} \tilde{q}_{\eta}{ }^{\alpha \eta \beta} g_{\alpha \beta}+g_{3} \sqrt{-g} .
$$

The matter Hamiltonian $\tilde{\mathcal{H}}_{\text {matter }}$ includes coupling of matter fields to curved space-time. The $(3,1)$ tensor density $\tilde{q}_{\eta}{ }^{\alpha \xi \beta}=\sqrt{-g} q_{\eta}{ }^{\alpha \xi \beta}$ is the affine momentum field mentioned above. The quadratic term endows space-time with kinetic energy and thus inertia and fundamentally modifies its dynamics.

The coupling constants $g_{1}, g_{2}$ and $g_{3}$ have the dimensions $\left[g_{1}\right]=1,\left[g_{2}\right]=L^{-2}$, and $\left[g_{3}\right]=L^{-4} \cdot g_{3}$ is usually identified with the vacuum energy density and gives rise to the socalled cosmological constant problem [50]. (Our conventions are the signature $(+,-,-,-$ ) of the metric and natural units $\hbar=c=1$. A comma before an index denotes partial derivative, and a semicolon denotes covariant derivative with the affine connection given by the gauge field. Pairs of indices in parentheses (brackets) denote (anti)symmetrization.)

The gauging process leads to the action integral

$$
S=\int d^{4} x \tilde{\mathcal{L}}=\int d^{4} x\left(\tilde{k}^{\alpha \nu \mu} g_{\alpha \nu ; \mu}-\frac{1}{2} \tilde{q}_{\alpha}{ }^{\beta \nu \mu} R_{\beta \nu \mu}^{\alpha}-\tilde{\mathcal{H}}_{\mathrm{Gr}}+\tilde{\mathcal{L}}_{\text {matter }}\right),
$$

where the total Lagrangian, a world scalar density, is split up into the modified gravity Lagrangian, displayed explicitly as a Legendre transform of the Hamiltonian $\tilde{\mathcal{H}}_{\text {Gr }}$ of Eq. (2), and the Lagrangian of matter, $\tilde{\mathcal{L}}_{\text {matter }}$, the Legendre transform of the yet unspecified $\tilde{\mathcal{H}}_{\text {matter }}$. In the integrand, the canonical nontensorial "velocity" of the affine connection, $\gamma_{\alpha \mu, \nu}^{\eta}$, as defined in the covariant de Donder formalism, is naturally substituted by the Riemann-Cartan tensor

$$
R_{\alpha \mu \nu}^{\eta}=\frac{\partial \gamma_{\alpha \nu}^{\eta}}{\partial x^{\mu}}-\frac{\partial \gamma_{\alpha \mu}^{\eta}}{\partial x^{\nu}}+\gamma_{\xi \mu}^{\eta} \gamma_{\alpha \nu}^{\xi}-\gamma_{\xi \nu}^{\eta} \gamma_{\alpha \mu}^{\xi}
$$

built from in general asymmetric affine connection coefficients. This is not an ad hoc substitution, but is the result of the requirement of diffeomorphism covariance implemented via the canonical transformation framework and the gauging process [44]. $\tilde{k}_{\alpha \beta v}$ is the metric momentum, the field conjugate to the metric $g_{\alpha \beta}$. It is by definition symmetric in its first two indices.

Variation of Eq. (3) with respect to the affine momentum gives the canonical equation

$$
R_{\beta v \mu}^{\alpha}=-2 \frac{\partial \tilde{\mathcal{H}}_{\mathrm{Gr}}}{\partial \tilde{q}_{\alpha}^{\beta v \mu}}
$$

\footnotetext{
2 When spinors are included, the tetrad field substitutes the metric as the fundamental field of the theory, and the spin connection emerges as a gauge field.

${ }^{3}$ Here, we also consider the constant term $g_{3} \sqrt{-g}$ that was omitted in [44].
} 
Its solution is for the Hamiltonian of Eq. (2)

$$
q_{\eta \alpha \xi \beta}=g_{1}\left(R_{\eta \alpha \xi \beta}-\hat{R}_{\eta \alpha \xi \beta}\right)
$$

where

$$
\hat{R}_{\eta \alpha \xi \beta}=g_{2}\left(g_{\eta \xi} g_{\alpha \beta}-g_{\eta \beta} g_{\alpha \xi}\right)
$$

is the Riemann curvature tensor of the maximally symmetric space-time, the (Anti) de Sitter geometry with the Ricci scalar curvature $12 g_{2}$. The affine momentum of space-time thus accounts for deformations of the space-time geometry relative to the (A)dS ground state.

Notice that the canonical field equation for the metric momentum,

$$
g_{\alpha \beta ; v}=\frac{\partial \tilde{\mathcal{H}}_{\mathrm{Gr}}}{\partial \tilde{k}^{\alpha \beta v}}=0,
$$

implements dynamically metric compatibility. This is due to the fact that the kinetic Hamiltonian $\tilde{\mathcal{H}}_{\mathrm{Gr}}$, Eq. (2), has been chosen to be independent of $\tilde{k}^{\alpha \beta v}$, the "metric momentum" conjugate to the metric tensor ${ }^{4}$.

The so-called consistency ("CCGG") equation that extends Einstein gravity $[43,44]$ is obtained as a combination of the canonical equations. It can be written as a local balance equation,

$$
-\Theta^{\mu \nu}=T^{\mu \nu}
$$

with

$$
\begin{gathered}
\Theta^{\mu \nu}:=\frac{2}{\sqrt{-g}} \frac{\partial \tilde{\mathcal{H}}_{\text {dyn }}}{\partial g_{\alpha \beta}} \\
T^{\mu \nu}:=\frac{2}{\sqrt{-g}} \frac{\partial \tilde{\mathcal{H}}_{\text {matter }}}{\partial g_{\alpha \beta}},
\end{gathered}
$$

and is similar to the stress-strain relation in elastic media. In analogy to the energy-momentum ("stress-energy") tensor of matter, $T^{\mu \nu}$, we interpret $\Theta^{\mu \nu}$ as the energy-momentum ("strainenergy") tensor of space-time ${ }^{5}$. Calculating now the strain-energy tensor (10) with the CCGG Hamiltonian (2), and substituting Eq. (5) for the momentum tensor, gives

$$
\Theta^{\mu \nu}=-g_{1} Q^{\mu \nu}+\frac{1}{8 \pi G}\left(G^{\mu \nu}+g^{\mu \nu} \lambda_{0}\right) .
$$

where

$$
G^{\mu \nu}:=R^{(\mu \nu)}-\frac{1}{2} g^{\mu v} R
$$

is the Einstein tensor ${ }^{6}$, and

$$
Q^{\mu \nu}:=R^{\alpha \beta \gamma \mu} R_{\alpha \beta \gamma}{ }^{\nu}-\frac{1}{4} g^{\mu \nu} R^{\alpha \beta \gamma \xi} R_{\alpha \beta \gamma \xi},
$$

\footnotetext{
${ }_{4}$ By Legendre transformation, $\tilde{k}^{\alpha} \beta v$ becomes then a Lagrange multiplier in the Lagrangian density.

5 This also implies that the total energy of the universe is zero, consistent with Jordan's conjecture, cf. [26]. Taking the vacuum expectation value of the "quantum analogue" of this equation, we would expect the vacuum energy densities of gravity and the matter to cancel each other, perhaps up to some residual value that can be identified with $g_{3} \approx 0$.

6 The Einstein tensor as derived from the canonical equations of motion contains only the symmetrized Ricci tensor. While in the absence of torsion the Ricci tensor is symmetric, it is not the case for nonzero torsion. The antisymmetric Ricci tensor interacts with the spin density of matter.
} 
is a trace-free, (symmetric) quadratic Riemann-Cartan concomitant. Equation (11) is a generalization of the 1.h.s. of the Einstein equation in three aspects. Firstly, the Palatini formalism is used, so the affine connection and the metric are still independent fields, torsion of space-time is admitted and a quadratic Riemann-Cartan term is added. In the Lagrangian constructed by Legendre transformation, that term is built from the Kretschmann scalar $R^{\alpha \beta \mu \nu} R_{\alpha \beta \mu \nu}$.

Notice that for aligning with the syntax used in GR, the coupling constants $g_{i}$ in Eq. (9) have been expressed in terms of the gravitational coupling constant $G$ and the cosmological constant $\lambda_{0}$ :

$$
\begin{aligned}
g_{1} g_{2} & \equiv \frac{1}{16 \pi G}=\frac{1}{2} M_{p}^{2} \\
6 g_{1} g_{2}^{2}+g_{3} & \equiv \frac{\lambda_{0}}{8 \pi G}=M_{p}^{2} \lambda_{0} .
\end{aligned}
$$

$M_{p}:=\sqrt{1 / 8 \pi G}$ is the reduced Planck mass. Combining the above equations yields

$$
\lambda_{0}=3 g_{2}+8 \pi G g_{3}=\frac{1}{M_{p}^{2}}\left(\frac{3 M_{p}^{4}}{2 g_{1}}+g_{3}\right),
$$

i.e., we find the cosmological constant being generated by the (A)dS curvature of the ground state of space-time, and the vacuum energy $g_{3}$ [49]. The parameter $g_{1}$ is the deformation parameter of the theory ${ }^{7}$ as it determines the strength of the quadratic Riemann-Cartan extension of Einstein gravity. (The coupling constant $g_{2}=M_{p}^{2} / 2 g_{1}$ is thus the inverse of the deformation parameter.)

\section{Geometrical stress energy and Cartan contortion density}

In the CCGG formalism, the tensors, $Q^{\mu v}$ and $G^{\mu v}$, are not necessarily covariantly conserved. That can even be the case if we set torsion to zero, as is known for Palatini-type theories with torsion not a priori excluded [5,21]. Indeed, straight algebra ${ }^{8}$ shows that

$$
Q_{\nu ; \mu}^{\mu} \equiv R_{; \mu}^{\alpha \beta \gamma \mu} R_{\alpha \beta \gamma \nu}+R^{\alpha \beta \gamma \mu}\left(R_{\alpha \beta \nu \tau} S_{\gamma \mu}^{\tau}-2 R_{\alpha \beta \gamma \tau} S_{\nu \mu}^{\tau}\right)
$$

where

$$
S_{\mu \nu}^{\lambda}=\frac{1}{2}\left(\gamma_{\mu \nu}^{\lambda}-\gamma_{\nu \mu}^{\lambda}\right)
$$

is Cartan's torsion tensor, vanishes for zero torsion and metric compatibility only if

$$
\bar{R}_{\alpha \beta \gamma}{ }^{\nu} \bar{\nabla}_{\mu} \bar{R}^{\alpha \beta \gamma \mu}=0 .
$$

The quantities with a bar above are calculated using the Christoffel symbol,

$$
\gamma_{\mu \nu}^{\lambda} \rightarrow\left\{\begin{array}{c}
\lambda \\
\mu \nu
\end{array}\right\}
$$

as for metric-compatible and torsion-free geometries the affine connection must be the Christoffel symbol uniquely determined by the metric via the Levi-Civita relation.

7 This result reminds of earlier approaches under the heading of de Sitter relativity to derive the cosmological constant and to explain cosmic coincidence and time delays of extra-galactic gamma-ray flares (see for example [1]).

8 We thank Julia Lienert for checking this identity with Maple. 
It is important to stress that the covariant conservation law for the strain-energy tensor, in Eq. (16), is not an identity, yet it facilitates, in addition to metric compatibility, a constraint linking the metric and the affine connection. Requirement (18) then restricts or even fixes the metric, and, in addition, implies that the r.h.s. of the consistency equation (9), the stress-energy tensor, is covariantly conserved, too.

Vice versa, requesting the stress-energy tensor of matter to be covariantly conserved, might in the CCGG theory lead to the necessity to adjust the affine connection with the given metric beyond the Levi-Civita relation. Physically, this opens a new channel within the dynamical geometry to take up its deformation energy, and this channel is based on torsion.

This can be illustrated as follows. If in the classical, macroscopic limit, torsion is neglected and the affine connection is assumed to be the Christoffel symbol, then Eq. (9) becomes

$$
g_{1}\left(\bar{R}^{\alpha \beta \gamma \mu} \bar{R}_{\alpha \beta \gamma}{ }^{\nu}-\frac{1}{4} g^{\mu \nu} \bar{R}^{\alpha \beta \gamma \delta} \bar{R}_{\alpha \beta \gamma \delta}\right)-\frac{1}{8 \pi G}\left[\bar{R}^{\mu \nu}-g^{\mu \nu}\left(\frac{1}{2} \bar{R}+\lambda_{0}\right)\right]=\bar{T}^{\mu \nu} .
$$

In order to explicitly work out the differences between the standard, GR-based cosmology and the CCGG model, we request the stress-energy tensor to be covariantly conserved:

$$
\bar{\nabla}_{\nu} \bar{T}^{\mu \nu}=0 \text {. }
$$

This is consistent as long as

$$
\bar{\nabla}_{\nu} \bar{\Theta}^{\mu \nu}=0
$$

holds, which for torsion-free geometries reduces to Eq. (18) for the Riemann tensor.

If a specific ansatz for the metric under the additional assumption of zero-torsion solves the "overbared" Eq. (20), but fails to satisfy constraint (18), the affine connection must not be Christoffel, though. With metricity in place, the most general form of the affine connection is

$$
\gamma_{\mu \nu}^{\lambda}=\left\{\begin{array}{c}
\lambda \\
\mu \nu
\end{array}\right\}+K_{\mu \nu}^{\lambda}
$$

with the contortion tensor 9

$$
K_{\lambda \mu \nu}=S_{\lambda \mu \nu}-S_{\mu \lambda \nu}+S_{v \mu \lambda}=-K_{\mu \lambda \nu} .
$$

The contortion tensor is a linear combination of the torsion tensor (17) and the metric. Obviously, deviating for a given metric from the Levi-Civita ansatz for the connection is equivalent to introducing torsion of space-time.

The Riemann-Cartan tensor can now be split into metric (Riemann) and torsion-dependent (Cartan) portions,

$$
R_{\alpha \beta \gamma \sigma}\left(\gamma_{\mu \nu}^{\lambda}\right) \equiv \bar{R}_{\alpha \beta \gamma \sigma}+P_{\alpha \beta \gamma \sigma}
$$

where

$$
P_{\lambda \sigma \mu \nu}:=\bar{\nabla}_{\mu} K_{\lambda \sigma v}-\bar{\nabla}_{\nu} K_{\lambda \sigma \mu}-K_{\lambda \beta \nu} K_{\sigma \mu}^{\beta}+K_{\lambda \beta \mu} K_{\sigma \nu}^{\beta}
$$

denotes the Cartan curvature tensor which is antisymmetric in the first and the second pairs of indices.

Then, with the definition of the symmetric $(2,0)$ tensor,

$$
\begin{aligned}
& \xi^{\mu \nu}(g, S):=\bar{Q}^{\mu \nu}-Q^{\mu \nu} \\
& \quad=\left(\frac{1}{4} g^{\mu \nu} \delta_{\sigma}^{\tau}-g^{\nu \tau} \delta_{\sigma}^{\mu}\right)\left(\bar{R}^{\alpha \beta \gamma \sigma} P_{\alpha \beta \gamma \tau}+\bar{R}_{\alpha \beta \gamma \tau} P^{\alpha \beta \gamma \sigma}+P^{\alpha \beta \gamma \sigma} P_{\alpha \beta \gamma \tau}\right),
\end{aligned}
$$

\footnotetext{
9 The generalization is to take also nonmetricity into account. The author of [35] considers a symmetric affine connection with nonmetricity.
} 
and the decomposition

$$
G^{\mu \nu}=\bar{G}^{\mu \nu}+P^{(\mu \nu)}-\frac{1}{2} g^{\mu \nu} P,
$$

the CCGG equation (20) is corrected to

$$
g_{1} \bar{Q}^{\mu \nu}-\frac{1}{8 \pi G}\left[\bar{G}^{\mu \nu}-g^{\mu \nu} \Lambda(x)\right]=\bar{T}^{\mu \nu}+\frac{1}{8 \pi G} P^{\prime(\mu \nu)}+g_{1} \xi^{\mu \nu} .
$$

The deviation from Eq. (20) is composed of terms that vanish with vanishing torsion ${ }^{10}$. Therefore,

$$
P^{\prime(\mu \nu)}=P^{(\mu \nu)}-\frac{1}{4} g^{\mu \nu} P
$$

is the trace-free Cartan-Ricci tensor. All tensors in this equation, including $\xi^{\mu v}$, are symmetric by definition.

When placing the tensors $P^{\prime(\mu \nu)}$ and $\xi^{\mu \nu}$ next to the stress-energy tensor of matter on the r.h.s. of the equation, it appears as a new, geometrical stress tensor. The geometrical stressenergy tensor is not covariantly conserved, and energy transfer from space-time is possible. Moreover, by its very definition, the term $P^{\prime(\mu \nu)}$ on the r.h.s. of Eq. (29) is trace-free, like the energy-momentum tensor of radiation or relativistic matter. Leaving only the Einstein tensor and the cosmological term on the 1.h.s. of the equation, i.e., taking the Einstein view of the system where all geometrical terms are considered as part of the extended, then covariantly conserved, stress-energy tensor, enables to study the newly emerging phenomena in relation to general relativity.

The Cartan-Ricci curvature scalar, $P(x)$, that we call Cartan contortion density as it is built from contortion and metric, is combined with the cosmological constant to the scalar cosmological field

$$
\Lambda(x):=\lambda_{0}+\frac{1}{4} P(x) .
$$

$\Lambda(x)$ reduces to a constant in torsion-free geometries and may not vanish even if the cosmological constant $\lambda_{0}$ does.

In the following, we demonstrate that under simplifying assumptions in the Friedman model, a unique solution of the cosmological field exists.

\section{The CCGG-Friedman model}

The Friedman model universe [22,51] is endowed with the Friedman-Lemaître-RobertsonWalker (FLRW) metric with curvature characterized by the parameter $K_{0}$,

$$
\mathrm{d} s^{2}=\mathrm{d} t^{2}+a^{2}(t)\left[\frac{\mathrm{d} r^{2}}{1-K_{0} r^{2}}-r^{2}\left(\mathrm{~d} \theta^{2}+\sin ^{2}(\theta) \mathrm{d} \varphi^{2}\right)\right] .
$$

The dimensionless parameter $a(t)$, the relative scale of the spatial section of the metric as function of the cosmological time $t$, remains the only dynamical freedom left. If $t_{0}$ is the current age of the universe, $a\left(t_{0}\right)=1$ applies to today. The parameter $K_{0}$ fixes the type of the underlying geometry: $K_{0}=0$ flat, $K_{0}>0$ spherical, $K_{0}<0$ hyperbolic.

With this one-parameter FLRW metric ansatz, Eq. (18) is satisfied only for three nonconstant solutions for the scale function $a(t)$ which, for cosmology, is in general too restrictive as it is independent of the matter content of the universe! Hence, Eq. (29) must be considered with the tensor corrections as outlined above. The torsion-induced tensor corrections on the

10 Notice that for an application in cosmology with just classical matter, the corresponding stress tensor is independent of the affine connection and hence is independent of torsion, $\bar{T}^{\mu \nu} \equiv T^{\mu \nu}$. 
r.h.s., $P^{\prime(\mu \nu)}$ and $\xi^{\mu \nu}$, must be diagonal, mimicking radiation and matter, respectively, with some unknown equations of state ${ }^{11}$. We accommodate schematically the tensor corrections to the stress-energy tensor by including cold dark matter in the density of dust and neglect radiation-like contributions (aka hot dark matter). Only the scalar Cartan contortion density in the cosmological field will be retained on the strain-energy side of the equation as a yet unknown dynamical quantity.

Due to the isotropy and homogeneity of the FLRW geometry, that cosmological field $\Lambda(x)$ can only depend on the universal time $t$. It formally corresponds to a density with the equation of state of dark energy ${ }^{12}$, and is therefore called here the dark energy function. The analysis is further simplified by adopting the scaling ansatz ${ }^{13}$

$$
\Lambda(t)=\Lambda(t(a))=: \Lambda_{0} f(a)
$$

with the dimensionless function $f(a)$, and the yet unspecified constant $\Lambda_{0}$.

The definitions and algebra leading to the Friedman equations are given in "Appendix A." The result is the modified Hubble function,

$$
H^{2}(a)=\sum_{i=r, m} C_{i} a^{-n_{i}}-\frac{k(a)}{a^{2}}+C_{\Lambda} f(a),
$$

where the geometrical effects emerging from the quadratic term have been combined with the curvature parameter $K_{0}$ of the FLRW metric to the curvature function ${ }^{14}$

$$
k(a):=K_{0}-\frac{\left(\frac{1}{4} C_{m} a^{-3}+C_{\Lambda} f(a)\right)\left(\frac{3}{4} C_{m} a^{-1}+C_{r} a^{-2}\right)}{\frac{1}{2} g_{2}-\frac{1}{4} C_{m} a^{-3}-C_{\Lambda} f(a)} .
$$

$k(a)$ is well defined since the function $f(a)$ obeys the unique differential equation derived in "Appendix B":

$$
\begin{aligned}
& \frac{\mathrm{d} f}{\mathrm{~d} a}=\frac{3 C_{m}}{4 C_{\Lambda}} a^{-4} \times \\
& \quad \frac{\frac{1}{2} g_{2}\left(\frac{3}{4} C_{m} a^{-3}+C_{r} a^{-4}\right)-\left(\frac{1}{2} g_{2}-\frac{1}{4} C_{m} a^{-3}-C_{\Lambda} f\right)\left(\frac{1}{4} C_{m} a^{-3}+C_{\Lambda} f\right)}{\frac{1}{2} g_{2}\left(\frac{3}{4} C_{m} a^{-3}+C_{r} a^{-4}\right)+\left(\frac{1}{2} g_{2}-\frac{1}{4} C_{m} a^{-3}-C_{\Lambda} f\right)^{2}} .
\end{aligned}
$$

By setting $g_{1}=0$ (which means $g_{2}=\infty$ ) and $f(a) \equiv 1$, we recover the Einstein-Friedman equation for the Hubble function based on General Relativity.

Moreover, as shown in "Appendix B," the modified Friedman equations cannot be solved with $f(a)=$ const., confirming the necessity to include the torsion corrections enforced

\footnotetext{
11 Whether these terms can explain the effect of (hot and cold) dark matter, and how they might impact cosmological perturbations, is a topic for separate studies.

12 The impact of the torsion-related corrections of the Einstein equation on cosmology has been discussed in [28]. Cosmology with a homogeneous spin density (aka Weyssenhoff fluid) was addressed in Refs. [11,12, 30,31,34,47]. A time-dependent cosmological constant has been also derived from string theory [3], and by the renormalization group method [29].

13 Under the assumption that $a(t)$ is a strictly monotonic function, $t(a)$ exists and is well defined. In the case of a bouncing or oscillating universe, though, care must be taken and the branches with $\dot{a}>0$ and $\dot{a}<0$ considered separately.

14 Treating the term invoked by the quadratic extension of the Hamiltonian as a curvature correction might seem arbitrary. However, its origin is the space-time side of the equation, and hence the other sensible option would be to combine it with the dark energy function. For an early analysis of that combination with a slightly different interpretation of the correction terms, see [49].
} 
by requiring concomitantly the FLRW metric and the covariant conservation of the energymomentum tensor of matter.

If we require the dark energy term to reproduce the observed present-day value of the cosmological constant and set $\Lambda_{0}=\Lambda_{\text {obs }}$, the initial condition $f(1)=1$ must hold. Similar reasoning for the curvature term gives $k(1)=k_{\mathrm{obs}}$, which implies

$$
k_{\mathrm{obs}} \equiv k(1)=K_{0}-\frac{\left(\frac{1}{4} C_{m}+C_{\Lambda}\right)\left(\frac{3}{4} C_{m}+C_{r}\right)}{\frac{1}{2} g_{2}-\frac{1}{4} C_{m}-C_{\Lambda}} .
$$

Setting $\Delta k:=K_{0}-k_{\mathrm{obs}}$, this relation can be resolved for $g_{2}$ giving

$$
\frac{1}{2} g_{2}(\Delta k)=\frac{1}{\Delta k}\left(\frac{1}{4} C_{m}+C_{\Lambda}\right)\left(\frac{3}{4} C_{m}+C_{r}+\Delta k\right) .
$$

With Eq. (14a), an equivalent expression for $g_{1}$ is obtained. Obviously, $g_{2}$ diverges and $g_{1}$ vanishes for $K_{0} \rightarrow k_{\text {obs. }}$. For a dark energy-dominated system, i.e., with $C_{m}=C_{r}=0$, Eq. (36) yields the solution $f(a) \equiv 1$. Then, Eq. (37) gives $\Delta k:=K_{0}-k_{\mathrm{obs}}=0$, and we obtain $g_{1}=0$. Hence, a dark energy-dominated CCGG universe is identical to the EinsteinFriedman model. Equation (34) is thus the equation of motion of the CCGG cosmology in the Einstein view.

The construction of the functions $f(a)$ and $k(a)$, and the selected boundary conditions for $a=1$, gives with Eq. (34) the Hubble constant

$$
H_{0}^{2}=\sum_{i=r, m, \Lambda} C_{i}-k_{\mathrm{obs}},
$$

which coincides with the standard value. Hence, we can adopt the parameters of the conventional Einstein-Friedman model based on the assumptions of almost flat FLRW metric, standard model of elementary particles, cold dark matter and a constant cosmological constant $\Lambda_{0}=\Lambda_{\text {obs }}$. (Concordance Model with the $\Lambda$ CDM parameter set as listed in Eq. (A.18) and Table 2.)

An important astronomical observable is also the dimensionless deceleration function

$$
q:=-\frac{\ddot{a}}{\dot{a}^{2}} a \equiv-\frac{\ddot{a}}{a} \frac{1}{H^{2}}=\frac{2 a^{2} M+V}{V+K_{0}},
$$

which explicitly depends on the curvature parameter $K_{0}$, and implicitly on the dark energy and curvature functions in the scale potential $V(a)$, Eq. (B.3). $M(a)$, viz. Eq. (A.17), is the Ricci scalar of the FLRW metric ${ }^{15}$. For the $\Lambda$ CDM parameter set, the present-day deceleration parameter $q_{0} \equiv q(1)$ is

$$
q_{0} \approx-0.5+K_{0} / H_{0}^{2} .
$$

Obviously, the value of both $K_{0} / H_{0}^{2}$ and $g_{1}$ is restricted by the measurement accuracy of $q_{0}{ }^{16}$. Recall that the parameter $K_{0}$ determines via Eq. (38) the coupling constant $g_{2}$, visualized in Fig. 1, and via $g_{1}=M_{p}^{2} / 2 g_{2}$ also the deformation parameter of the theory.

15

$$
R^{F L R W}=6\left[\frac{\ddot{a}}{a}+\left(\frac{\dot{a}}{a}\right)^{2}+\frac{K_{0}}{a^{2}}\right]=3 C_{m} a^{-3}+4 \Lambda(a)=12 M(a)
$$

16 For a discussion, see, for example, [8,32]. 


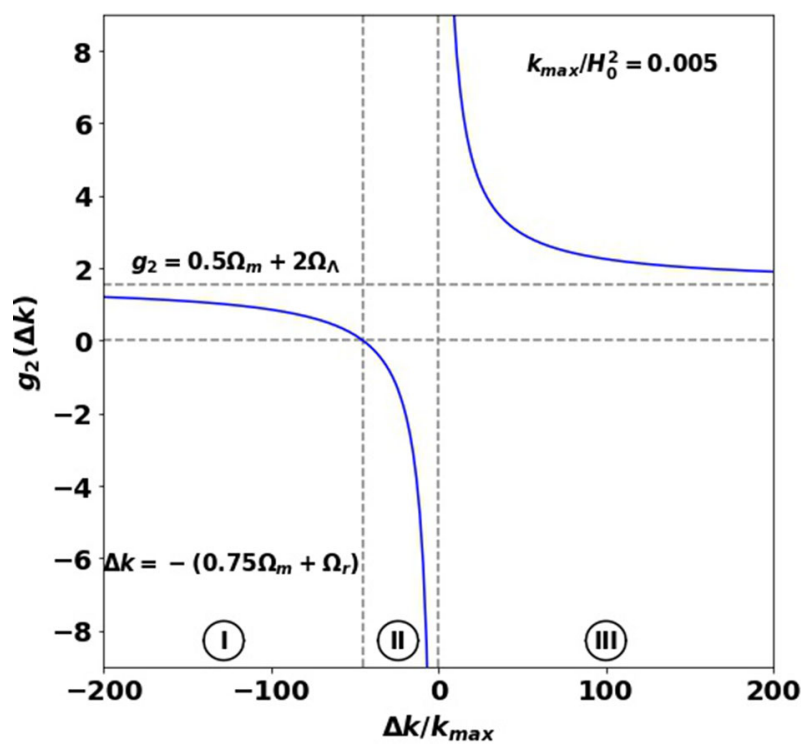

Fig. 1 The relation $g_{2}(\Delta k)$ is displayed in units $H_{0}^{2}$. As $g_{2}$ must be nonzero and finite in order to maintain the consistency of the theory, the root $\Delta \bar{k} \equiv-3 C_{m} / 4-C_{r}$, where $g_{2}=0$, and $\Delta k=0$ with $g_{2} \rightarrow \pm \infty$ are both "forbidden" values. For $\Delta k \rightarrow \pm \infty$, we find $g_{2} \rightarrow C_{m} / 2+2 C_{\Lambda}$

It is important to stress at this point that $K_{0}=k_{\mathrm{obs}}$ is possible if and only if $g_{1}=0$, i.e. only in the realm of the Einstein-Friedman model. The limiting process, $g_{1} \rightarrow 0$, is continuous but not convergent, though, since $g_{2} \sim 1 / g_{1}$ diverges there! $!^{17}$.

Obviously, for this type of analysis and within the present accuracy of observations, the CCGG-Friedman model with the six priors of the Concordance Model is a reasonable ansatz.

\section{Scenario analysis}

In this section, we investigate the impact of the remaining free parameter, the curvature $K_{0}$ of the Friedman metric, on the evolution of the universe. We follow the standard practice and set $C_{k}=-k_{\mathrm{obs}}=0$, i.e., we accept the present universe to be with a high accuracy flat for consistency with the observed CMB radiation isotropy [32]. Then, $\Delta k \equiv K_{0}$. We vary the parameter $K_{0} / k_{\max } \in \mathbb{R}$ that enters the formula for the calculation of the coupling constant $g_{2}$ - and the deformation parameter $g_{1}$. In order to remain close to the Concordance Model, we vary $K_{0}$ on the scale of $k_{\max }$ inferred from observations, namely $\left|k_{\mathrm{obs}}\right| \leq k_{\max }=0.005 H_{0}^{2}$, cf. Eq. (A.18b). The values of $K_{0}, g_{2}$ (and $\left.g_{1}\right)$ applied in the following calculations are listed in Table 1.

For a given $K_{0}$, we calculate $g_{2}$ from Eq. (38) and then numerically solve the differential equation (36) for $f\left(a ; g_{2}\left(K_{0}\right)\right)$ using the six priors of the Concordance Model. The dynamics

\footnotetext{
17 That would give rise to an inconsistency as the quadratic term then diverges in the Hamiltonian while it vanishes in the Lagrangian. $g_{1}$ and $g_{2}$ must thus be finite in order for the Hamiltonian theory to be consistent with the action principle. The dimensionless deformation parameter $g_{1}$ appears as the measure of the inertia of space-time in line with the formal rôle of the Riemann-Cartan tensor as the covariant "velocity" of the affine connection field.
} 
Table 1 The table lists the values of the parameter $K_{0} / k_{\max }$ used alongside the default $\Lambda \mathrm{CDM}$ parameter set in the following calculations. Three combinations with the sign of the pertinent coupling constant $g_{2}\left(K_{0}\right)$ (in units $H_{0}^{2}$ ) and the deformation parameter $g_{1}$ (dimensionless) yield three parameter Regions I (top), II (middle) and III (bottom). The values $g_{2}=0, \pm \infty$ are limiting cases outside the realm of the CCGG theory. $k_{\max } \equiv+0.005 H_{0}^{2}$

\begin{tabular}{|c|c|c|}
\hline$K_{0} / k_{\max }$ & $g_{2} / H_{0}^{2}$ & $g_{1}$ \\
\hline \multicolumn{3}{|l|}{ Region I } \\
\hline-80 & 0.66 & $1.99 \times 10^{120}$ \\
\hline-70 & 0.54 & $2.45 \times 10^{120}$ \\
\hline-60 & 0.37 & $3.56 \times 10^{120}$ \\
\hline-46 & 0.03 & $3.85 \times 10^{121}$ \\
\hline \multicolumn{3}{|l|}{ Excluded } \\
\hline-45 & 0.00 & $\pm \infty$ \\
\hline \multicolumn{3}{|l|}{ Region II } \\
\hline-44 & -0.04 & $-3.68 \times 10^{121}$ \\
\hline-30 & -0.78 & $-1.67 \times 10^{120}$ \\
\hline-10 & -5.43 & $-2.39 \times 10^{119}$ \\
\hline-1 & -68.21 & $-1.90 \times 10^{118}$ \\
\hline \multicolumn{3}{|l|}{ Excluded } \\
\hline 0.00 & $\pm \infty$ & 0.00 \\
\hline \multicolumn{3}{|l|}{ Region III } \\
\hline 1 & 71.31 & $1.82 \times 10^{118}$ \\
\hline 10 & 8.53 & $1.52 \times 10^{119}$ \\
\hline 45 & 3.10 & $4.18 \times 10^{119}$ \\
\hline 80 & 2.42 & $5.35 \times 10^{119}$ \\
\hline
\end{tabular}

of the universe's expansion is thereby determined by the equation

$$
\dot{a}^{2}+V(a)=-K_{0},
$$

derived in "Appendix B," cf. Eq. (B.2). It is formally the total energy of a fictitious classical, nonrelativistic point particle with mass 2 , moving with the velocity $\dot{a}$, in the one-dimensional potential $V(a)$. Having the total energy $-K_{0}$, that particle will accelerate when "sliding down" the potential wall until it hits the minimum, and decelerate when climbing up its walls as long as its kinetic energy remains positive. The equation $V\left(a_{i}\right)=-K_{0}$ thus determines a turning point that corresponds to a bounce of the universe at a (possibly finite) extension scale $a_{i}$. The area $\left\{a: V\left(a ; g_{2}\left(K_{0}\right)<-K_{0}\right\}\right.$ is a "forbidden zone" ${ }^{18}$. Notice that the equation of motion (42) is time-reversal invariant, so if $\dot{a}$ is a solution, then also $-\dot{a}$, and expansion and contraction are in principle equally possible. The two branches can join continuously at the point $a$ where $\dot{a}=0$.

In order to check the validity of the energy conditions [13] in the Einstein view, the effective total energy density and pressure must include matter, radiation, dark energy and other geometry-driven terms. They, and the resulting EOS, are derived from Eqs. (A.5) and (A.6), using the definitions $\Omega_{i} \equiv C_{i} / H_{0}^{2}$ and $\rho_{\text {crit }} \equiv 3\left(H_{0} M_{p}\right)^{2}$ :

$$
\rho(a):=\rho_{\text {crit }}\left(\Omega_{m} a^{-3}+\Omega_{r} a^{-4}+\Omega_{\Lambda} f(a)-\frac{k(a)}{H_{0}^{2} a^{2}}\right)=\rho_{\text {crit }} H^{2}(a) / H_{0}^{2},
$$

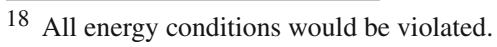




$$
\begin{aligned}
& p(a):=\rho_{\text {crit }}\left(\frac{1}{3} \Omega_{r} a^{-4}-\Omega_{\Lambda} f(a)-\frac{1}{3} \frac{k(a)-2 K_{0}}{H_{0}^{2} a^{2}}\right), \\
& \omega(a):=\frac{p(a)}{\rho(a)}=\frac{1}{3}[2 q(a)-1] .
\end{aligned}
$$

Both the total density and pressure reduce to the standard Einstein-Friedman expressions with $f(a) \equiv 1$ and $g_{1}=0$. Notice that the conditions NEC, WEC and DEC are satisfied if $\rho(a) \geq 0$ and $\omega(a) \geq-1$ hold. For SEC to be met, $\omega(a) \geq-\frac{1}{3}$ is required.

The role of the parameter $K_{0}$ is intricate as its value determines implicitly-via the coupling constant $g_{2}$ - the shape of the scale potential. Its physical impact is analyzed in the following sections for the Regions I $\left(K_{0}<\bar{K}_{0}\right)$, II $\left(\bar{K}_{0}<K_{0}<0\right)$ and III $\left(K_{0}>\bar{K}_{0}\right)$, separated by the critical values $\bar{K}_{0}=-\frac{3}{4} C_{m}+C_{r} \approx-45.011 k_{\max }=0.22506 H_{0}^{2}$ for the Default parameter set (cf. Table 2), and $K_{0}=0$. Recall that $\bar{K}_{0}$ is critical as for this value $g_{2}$ vanishes and the deformation parameter $g_{1}$ is infinite, which means that the CCGG equation is dominated by the scale-invariant quadratic gravity. For $K_{0}=0$, on the other hand, the deformation parameter $g_{1}$ vanishes, corresponding to the pure Einstein gravity limit. The deformation parameter thus interpolates the theory between the scale-invariant and the Einstein-Hilbert versions. The Lagrangians for both limits exist but outside of the domain of the CCGG theory.

A remark is due on the relation of the curvatures $K_{0}$ vs. $k(a)$. The former is the constant parameter of the FLRW metric, and the latter is the CCGG curvature function. Once $K_{0}$ is inferred from observations (e.g., of $\left.q_{0}\right)$, then the function $k\left(a ; K_{0}\right)$ is fixed. On the other hand, analyses of observations based on Einstein gravity will, at any given past or future instant $t_{f}$, misinterpret the value of $k\left(a\left(t_{f}\right)\right)$ as a (possibly time-dependent) FLRW curvature parameter, albeit the space-time geometry is defined with $K_{0}$.

We note in passing that we carry out the calculation with a high-precision toolkit ${ }^{19}$ to provide an initial evaluation and interpretation of the emerging dynamical scenarios, even though for very small-scale parameters $a \rightarrow 0$ both the physics and the numerics of the results may be questioned. We leave the ultimate judgment on the physical significance of the results to a more comprehensive analysis.

\subsection{Region I: Nonsingular Bounce with steady inflation}

In this case, $K_{0}<\bar{K}_{0}$ and both $g_{2}$ and $g_{1}$ are finite and positive. The scale potentials $V\left(a ; g_{2}\left(K_{0}\right)\right)$, Eq. (B.3), for the various negative values of the parameter $K_{0}<\bar{K}_{0}$ are plotted in Fig. 2.

The potential becomes greater than the total energy $-K_{0}$ at some turning point $a_{i}$. As the dynamics is time-reversal invariant, a possible shrinking of the universe comes to a sudden hold at $a_{i}$ and is smoothly reversed ${ }^{20}$ to an expansion, resulting in a nonsingular bounce. That dynamics is summarized in the phase-space plot in Fig. 3. Notice that with $K_{0} \rightarrow \bar{K}_{0}$ the turning point $a_{i}$ moves to smaller scales and larger redshifts $z$. This corresponds to $g_{1} \rightarrow \infty$, i.e., to an increasing impact of quadratic gravity.

Unlike in Einstein-Friedman cosmology, the space-time in CCGG acquires kinetic energy when deformed from the de Sitter $^{21}$ ground state. The contribution of that kinetic energy to

\footnotetext{
19 All calculations were performed using mpmath, a Python library for floating point arithmetic with arbitrary precision. [25]

20 The expansion continues though for ever. The theory as is does not implicate a reversal (at time $t=\infty$ ?) of that expansion

${ }^{21}$ Its Ricci scalar is positive, $g_{2}>0$.
} 


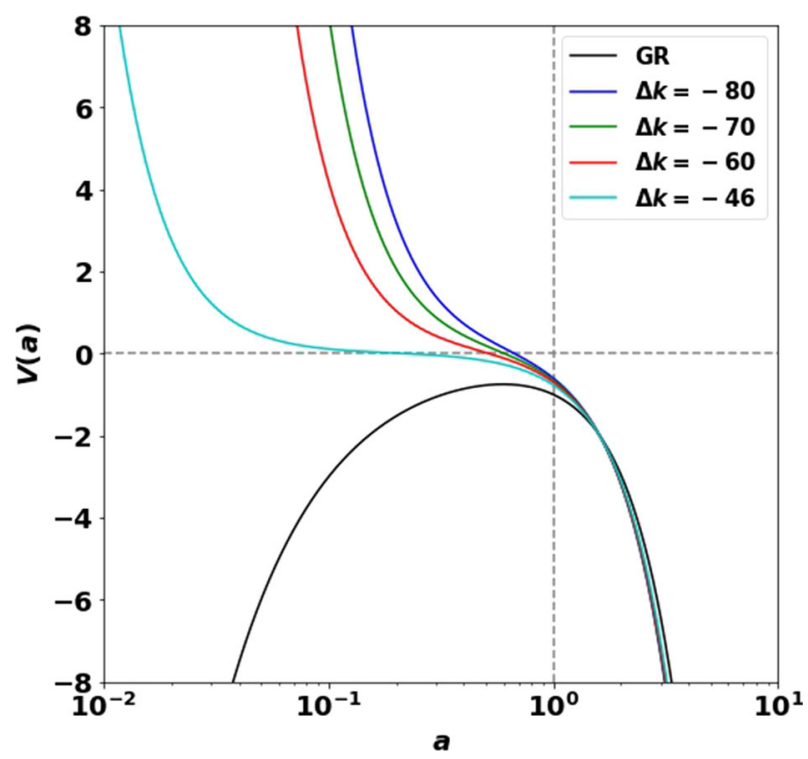

Fig. 2 Log-lin plot of the scale potentials $V\left(a ; g_{2}\left(K_{0}\right)\right)$ in units of $H_{0}^{2} . V+K_{0}>0$ is the forbidden region of scale expansion. The curve labeled GR shows the potential of the conventional, flat Einstein-Friedman cosmology where $f(a) \equiv 1, g_{1}=0 . K_{0}=\Delta K \cdot 0.005 H_{0}^{2}$

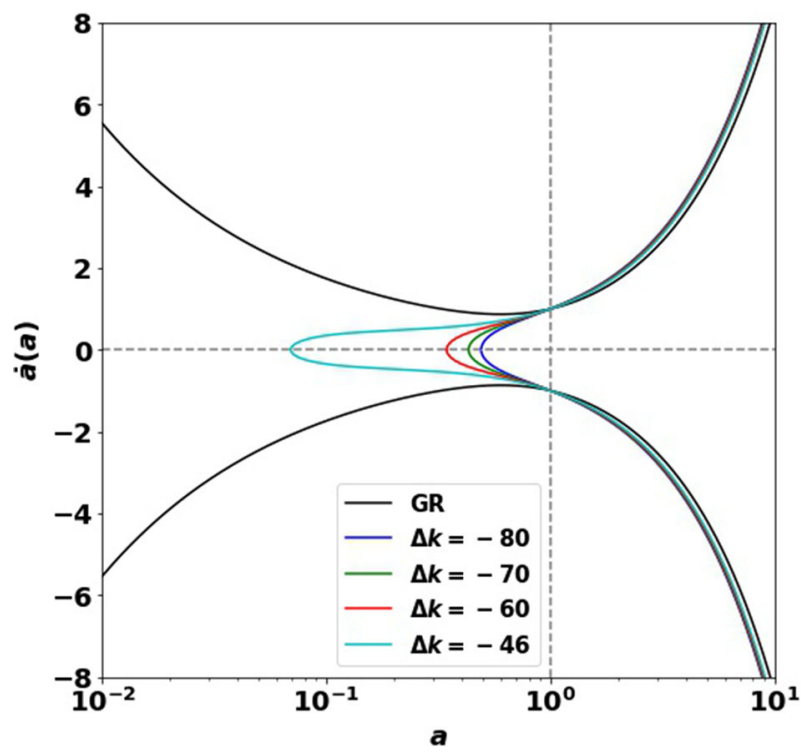

Fig. 3 "Phase space" plot of the expansion rate $\dot{a}$ (in units $H_{0}$ ) versus the scale parameter $a$. The transition from collapse (lower branch with $\dot{a}<0$ ) to expansion (upper branch with $\dot{a}>0$ ) is smooth implying a hold at a finite size (nonsingular, soft bounce)

the overall energy balance increases with increasing deformation of space-time, and also with increasing "inertia of space-time" $g_{1}$. Due to the requirement of covariant conservation of the energy-momentum tensor of ordinary matter, it is stored in the form of contortion density, 


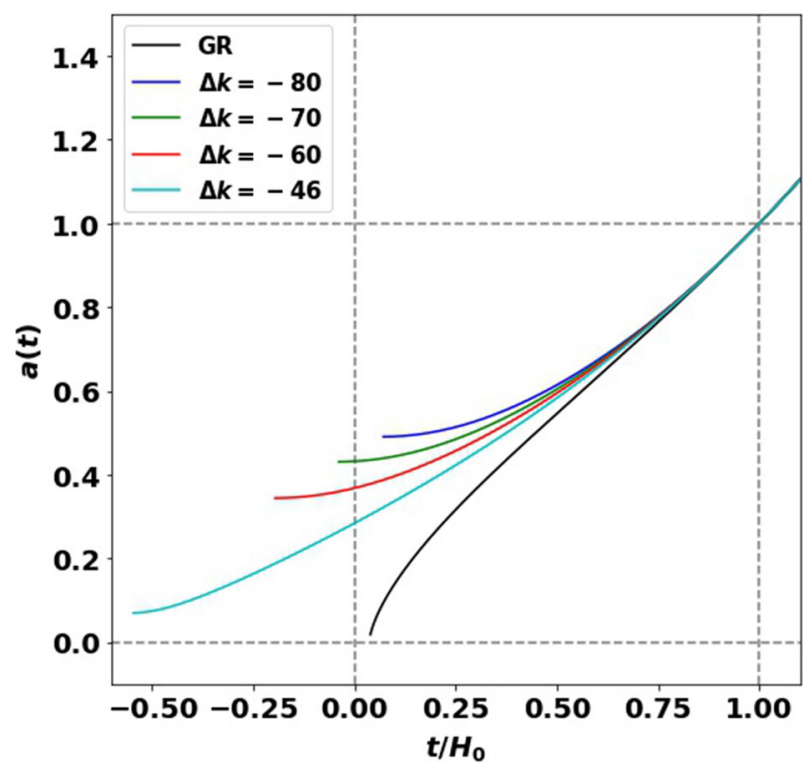

Fig. 4 Lin-lin plot of the expansion scale as a function of the universal time. It is finite at the time of birth of the universe that occurs at $a_{i} \equiv a\left(t_{i}\right)>0$ and $\dot{a}\left(t_{i}\right)=0$. GR refers to the Einstein-Friedman cosmology

aka dark energy, and in the curvature correction that in the Einstein view of the CCGG theory contributes to the (geometric) stress-energy tensor. These are the energy sources driving the inflation.

The conventional scale potential of the flat Einstein-Friedman cosmology (labeled GR), with just the standard (dark) matter and radiation terms and a constant cosmological constant, does not lead to inflation in the early universe. On the other hand, the scale potentials derived from CCGG display both inflationary behavior and graceful exit to the dark energy era of GR in the late epoch $(a \gg 1)$. The graceful exit occurs as the corrections pertinent to the CCGG theory become constant, asymptotically converging to the GR values. (For details of the various contributions to the Hubble function and of the energy conditions, see "Appendix C.1." Since $\omega<-1$ in the early epoch, all energy conditions are violated.)

The scale parameter as a function of the universal time, $a(t)$, is given by the integral

$$
t-t_{i}=\int_{a_{i}}^{a}\left[-K_{0}-V\left(a^{\prime} ; f\left(a^{\prime} ; K_{0}\right)\right)\right]^{-1 / 2} d a^{\prime},
$$

derived from Eq. (B.2) where the solution $f\left(a ; K_{0}\right)$ of Eq. (36) is inserted. We fix the global timescale by setting $t(1)=1 / H_{0}$. Then, $\dot{a}(t)$ is given by inserting $a(t)$ (cf. Fig. 4) into $\sqrt{-K_{0}-V(a)}$. While the so-calculated expansion rate at time $t_{i}$ vanishes, i.e. $\dot{a}\left(t_{i}\right)=0$, the acceleration is positive there, $\ddot{a}\left(t_{i}\right)>0$, as shown in the plot in Fig. 5. The universe thus evolves from a nonsingular, soft bounce ("Little Bang") through an everlasting inflation, with phases of varying intensity, and finally, via a graceful exit into the dark energy era. The initial scale $a_{i}$ of the universe decreases with $g_{2} \rightarrow 0$, while its age and the violence of the explosion, $\ddot{a}_{i}$, increase. The "soft" characteristic, namely $\dot{a}\left(t_{i}\right)=0$, of this nonsingular bounce scenario is maintained, though.

The scenario thus encountered in the parameter Region I has the following characteristic:

1. No singularity - the universe starts off from a finite volume, 


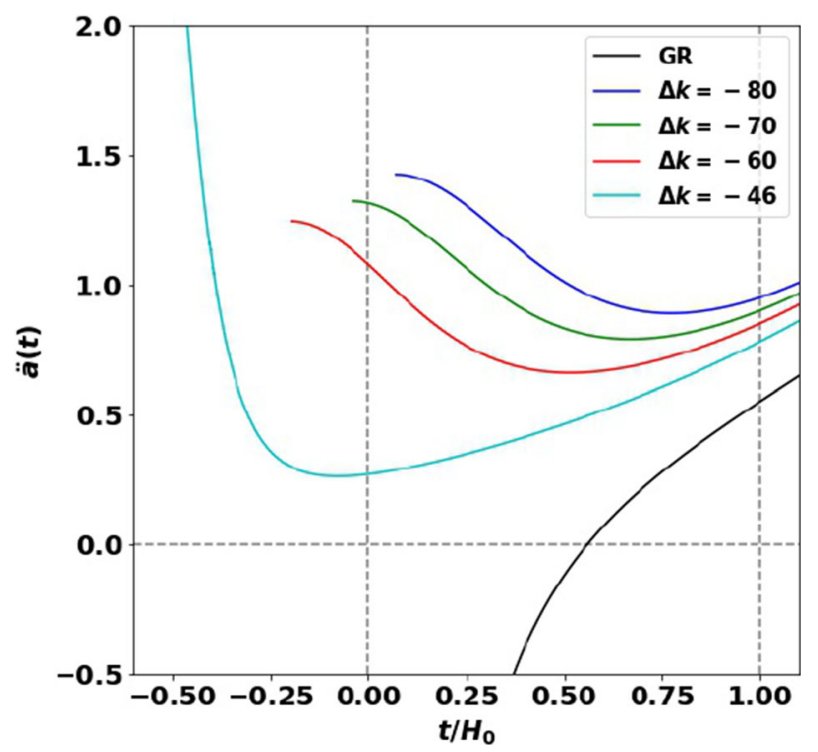

Fig. 5 Acceleration $\ddot{a}(t)$ (units $H_{0}^{2}$ ) as a function of the universal time $t$. It is always positive indicating an everlasting inflation of the universe. For $K_{0} \gtrsim-70$, the age of the universe exceeds the age inferred from Einstein gravity

2. Soft bounce with initial zero expansion rate but nonzero acceleration,

3. Steady inflation commencing after the bounce,

4. "Graceful exit" to the late dark energy era, similar to Einstein gravity.

We call it the Nonsingular Bounce scenario.

5.2 Region II: Hard singular Big Bang with a secondary inflation-deceleration phase

In this case, $0>K_{0}>\bar{K}_{0}$, and both $g_{2}$ and $g_{1}$ are finite and negative (AdS geometry), i.e., the kinetic energy from the quadratic term in the Hamiltonian has the opposite sign compared to Region I. With $K_{0} \rightarrow \bar{K}_{0}$, the limiting value for the coupling constant is $g_{2} \rightarrow 0$, i.e., equivalently $g_{1} \rightarrow-\infty$ which means a dominant quadratic gravity. The scale potentials, plotted as $-K_{0}-V\left(a ; g_{2}\left(K_{0}\right)\right)$ in Fig. 6, differ from the GR-scale potential but never exceed the "total energy" $-K_{0}$.

Moreover, the asymptotic behavior, $V(a \rightarrow 0)=-\infty$, is similar to Einstein cosmology, and we expect this type of universe to be singular. Indeed, the phase-space plot in Fig. 7 confirms this expectation. Interpreting the phase-space diagram as if the birth of the universe had occurred after a Big Crunch, it resembles a supernova explosion where the kinetic energy of the collapsing matter drives the subsequent explosion.

Unlike the nonsingular Bounce scenario with negative $K_{0}$ and positive $g_{2}$ discussed in Sect. 5.1, the initial acceleration at time $t_{i}$ is negative. It changes sign though at $a \sim 10^{-3}$. The following inflation-deceleration phase is depicted in Fig. 8. The initial expansion thus slows down and terminates at the first peak of the potential, with a secondary inflation that terminates when the scale hits the bottom of the potential well [4], after which a second deceleration phase commences. 


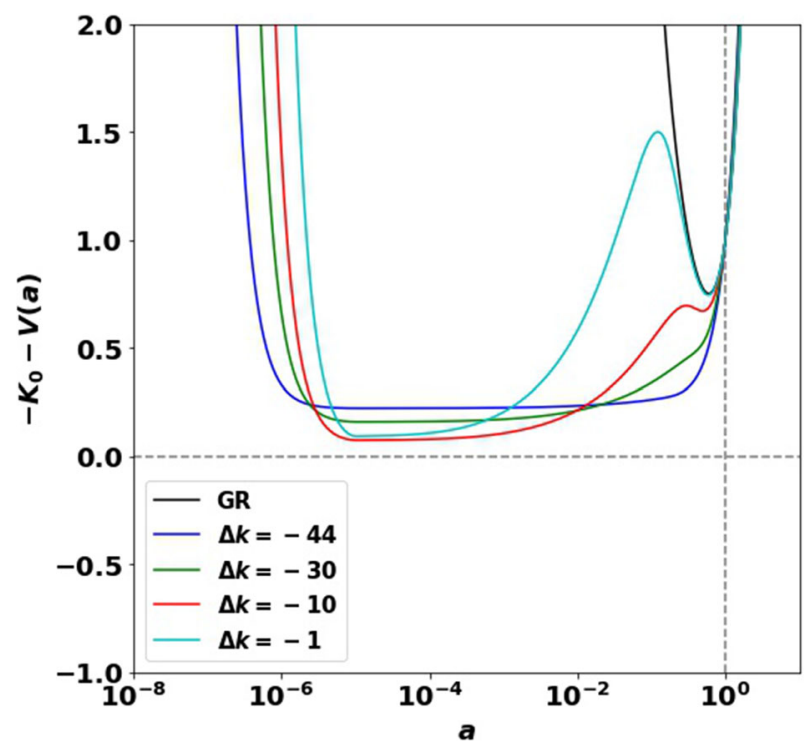

Fig. 6 Scale potentials $-K_{0}-V\left(a ; g_{2}\left(K_{0}\right)\right)$ in units of $H_{0}^{2}$. Since $-K_{0}-V\left(a ; g_{2}\left(K_{0}\right)\right)>0$ always holds, there is no turning point. The curve labeled GR is the scale potential of the conventional flat Einstein-Friedman $\operatorname{cosmology}\left(f(a) \equiv 1, g_{1}=0\right)$

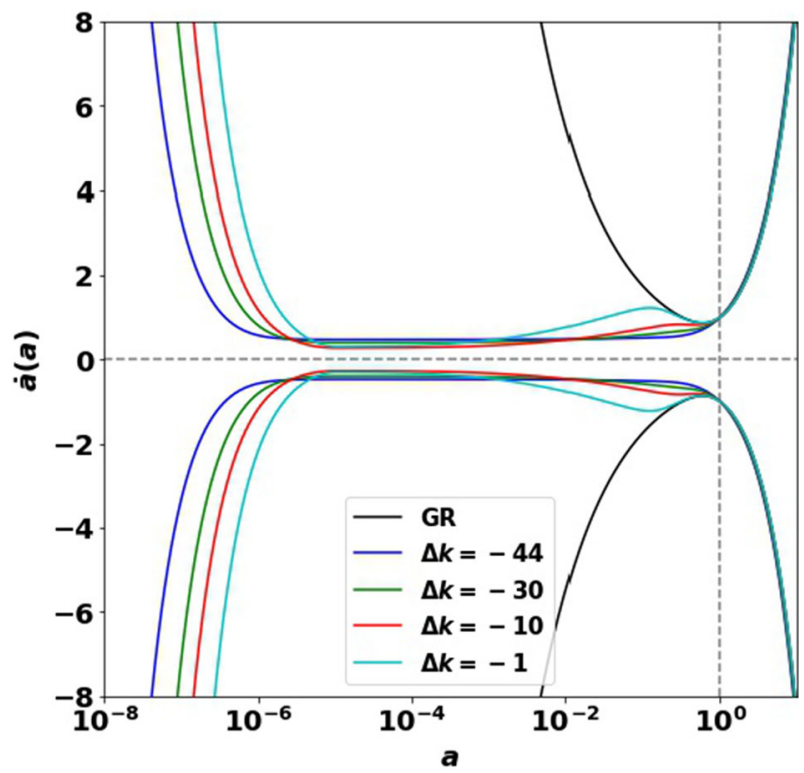

Fig. 7 "Phase space" plot of the expansion rate $\dot{a}$ (in units $H_{0}$ ) vs. the scale parameter $a$. The expansion phase with $\dot{a}>0$ is displayed in the upper panel, while the lower panel corresponds to a collapse toward $a=0$

That deceleration stops at the top of the second peak of the scale potential, followed by soft re-acceleration that reaches the value $q_{0}=-0.5+K_{0} / H_{0}^{2}$ today and continues into the dark energy era. The corresponding deceleration parameter $q(a)$, see Fig. 9, illustrates the 


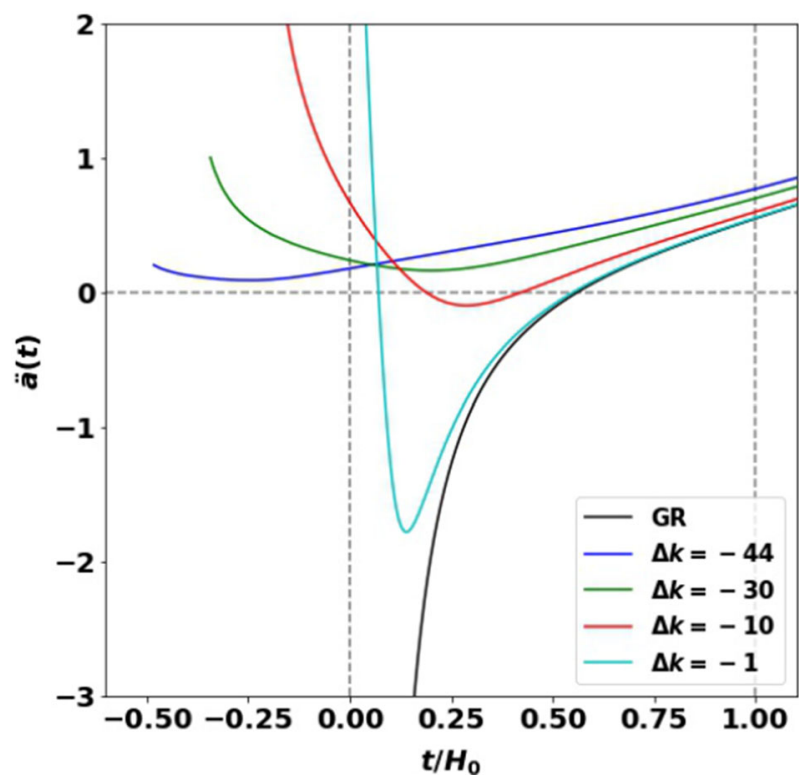

Fig. 8 Acceleration $\ddot{a}(t)$ (units $H_{0}^{2}$ ) as a function of the universal time $t$. The acceleration of the inflation phase goes over into a deceleration period with $\ddot{a}(t)<0$ and rebounds again at a lower level in the late era

acceleration-deceleration transition in more detail. Its present-day value decreases linearly with $K_{0}$. In Ref. [36], the most recent deceleration-acceleration transition has been identified at redshift in the region $z^{*} \approx 0.3-0.7$, which corresponds to the scale $a^{*}=1 /\left(1+z^{*}\right) \approx$ $0.6-0.8$. The authors of Ref. [36] also conjecture that the cosmological constant must be time dependent. Notice that the local extrema of the potential become more pronounced and shift to smaller scales and earlier times with $K_{0} \rightarrow 0_{-}$.

The singularity of the Big Bang is illustrated in the plot in Fig. 10 where the scale parameter is shown to asymptotically reach zero at some finite time $t_{i}\left(K_{0}\right)$, in general exceeding $H_{0}^{-1}$. The "hard" character of the Big Bang is attributed to the initial non-zero expansion rate, cf. Fig. 11.

A Singular "Hard" Big Bang scenario thus emerges in the parameter Region II. It is initially similar to Einstein-Friedman cosmology but with a "built-in" inflation phase. It has the following characteristic:

1. Singularity $a\left(t_{i}\right)=0$,

2. Violent initial expansion with diminishing acceleration, similar to conventional Big Bang, and comparable to a supernova explosion,

3. A secondary inflation-deceleration phase,

4. Graceful exit into a second late inflation epoch (dark energy era) at $a^{*} \approx 0.6$.

The overall behavior of the scale expansion is again driven by the interplay of the correction caused by the dynamics of the space-time geometry leading to a negative effective pressure, see "Appendix C.1." Notice that all energy conditions are satisfied except SEC. 


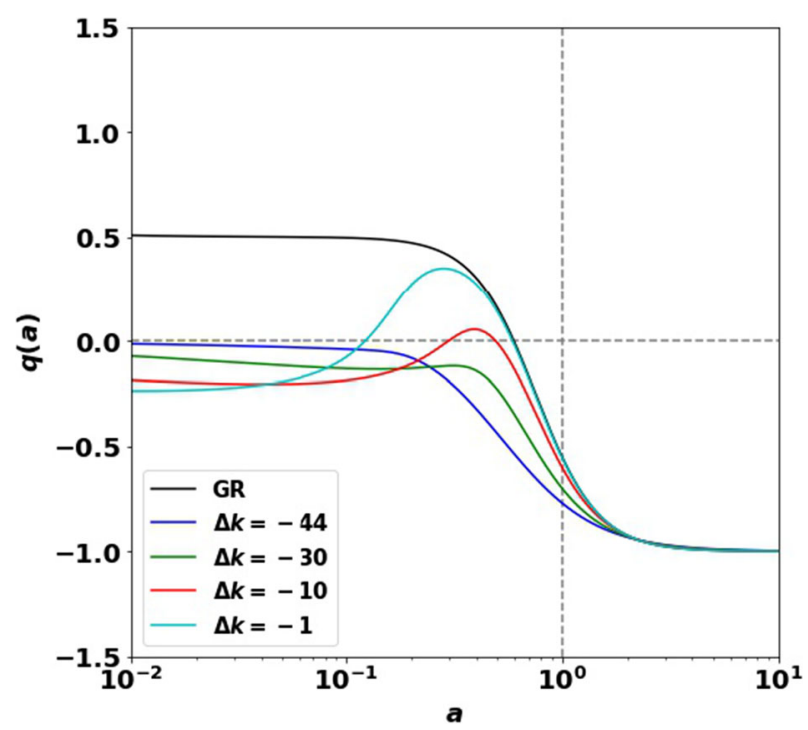

Fig. 9 Log-lin plot of the dimensionless deceleration parameter $q\left(a ; K_{0}<0\right)$ showing the region of the most recent transition from deceleration to acceleration of the expansion of the universe. The transition scale $a^{*}$ is defined by $q\left(a^{*}\right)=0$

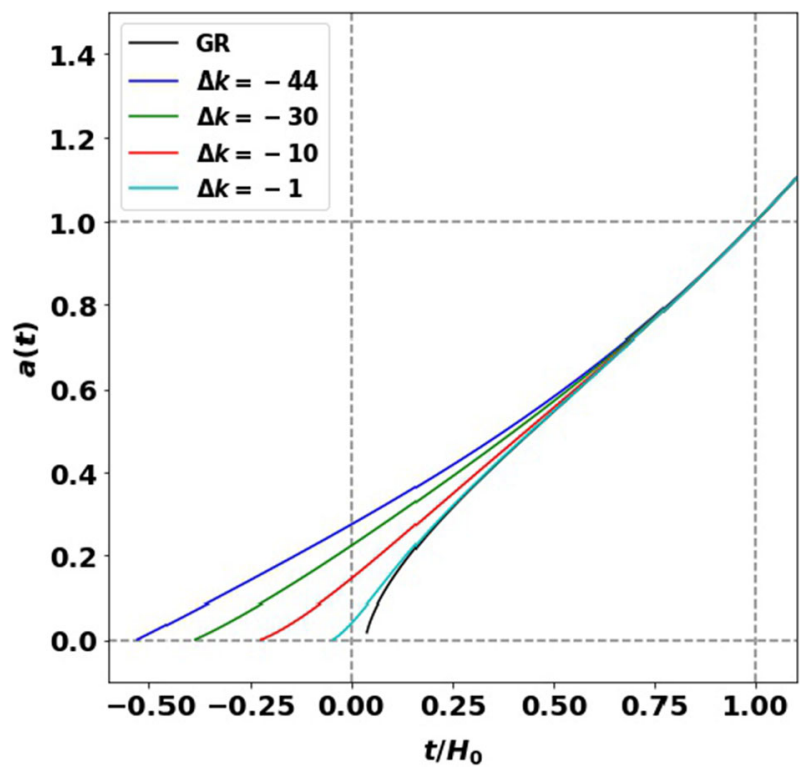

Fig. 10 Lin-lin plot of the scale parameter as a function of the universal time indicating a singular Big Bang and a finite age of the universe. GR refers to Einstein gravity

\subsection{Region III: Singular hard Big Bang without inflation}

In the parameter Region III, $K_{0}>0$ and both $g_{2}$ and $g_{1}$ are finite and positive. For $K_{0} \rightarrow 0$, the limiting value for the coupling constant is $g_{2} \rightarrow \infty$ (and equivalently $g_{1} \rightarrow 0$ ). The resulting 


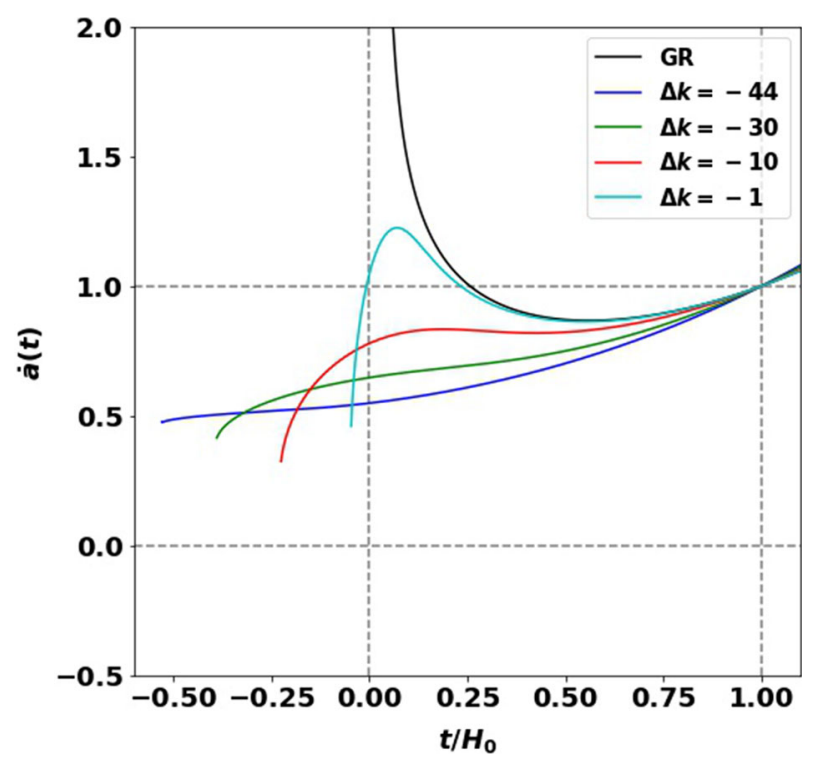

Fig. 11 Expansion rate $\dot{a}(t)$ (units $H_{0}$ ) commences with a nonzero value and diminishes as a function of the universal time

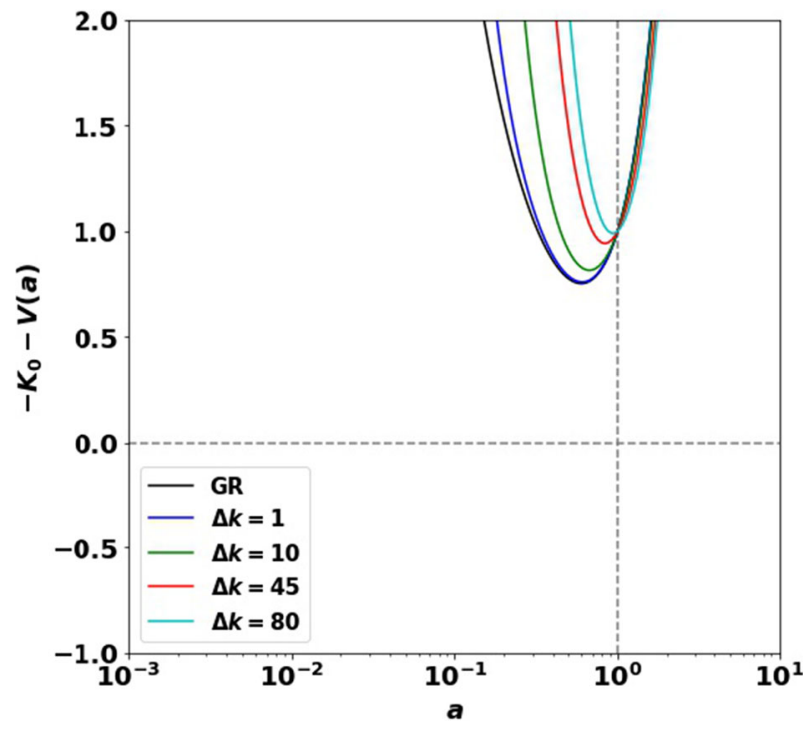

Fig. $12 \log -$ lin plot of the scale potentials $-K_{0}-V\left(a ; g_{2}\left(K_{0}\right)\right)$ in units of $H_{0}^{2}$. The curve labeled GR shows the potential of the conventional flat Einstein-Friedman theory $\left(f(a) \equiv 1, g_{1}=0\right)$

scale potentials, displayed in Fig. 12, give rise to a singular Big Bang scenario as in Einstein gravity. The Einstein-Friedman scenario is asymptotically approached with $\Delta k=K_{0} \rightarrow 0$ as seen also from the phase-space plot in Fig. 13.

The singularity and the violent character of the Big Bang are obvious as $a\left(t_{i}\right)=0$ and $\ddot{a}\left(t_{i}\right) \neq 0$, cf. the plots in Figs. 14 and 15. The acceleration is negative in the early phase; hence, there is no inflation caused by the dynamical space-time geometry. The character of the 


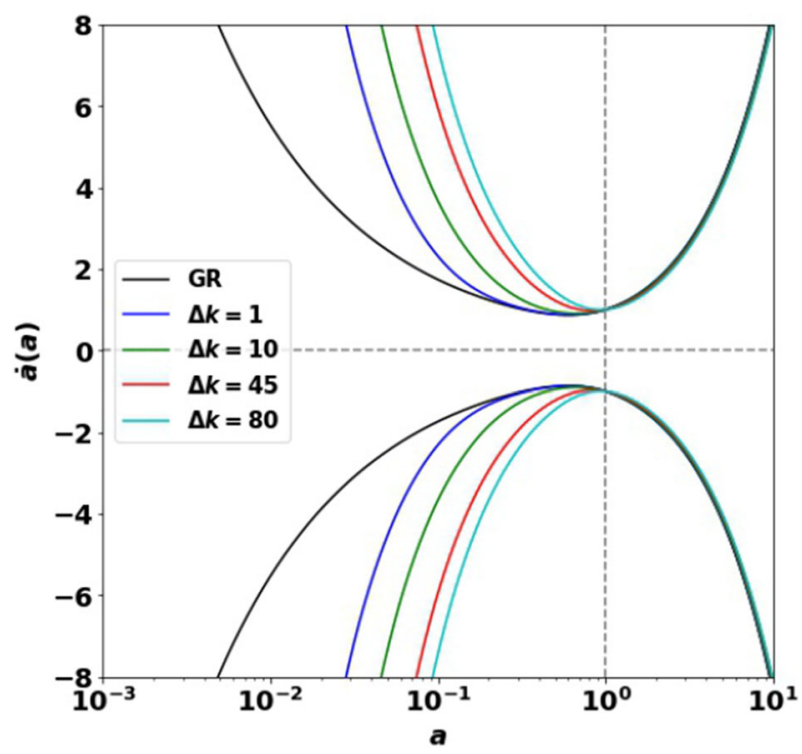

Fig. 13 "Phase space" plot of the expansion rate $\dot{a}$ (units $H_{0}^{2}$ ) versus the scale parameter $a$

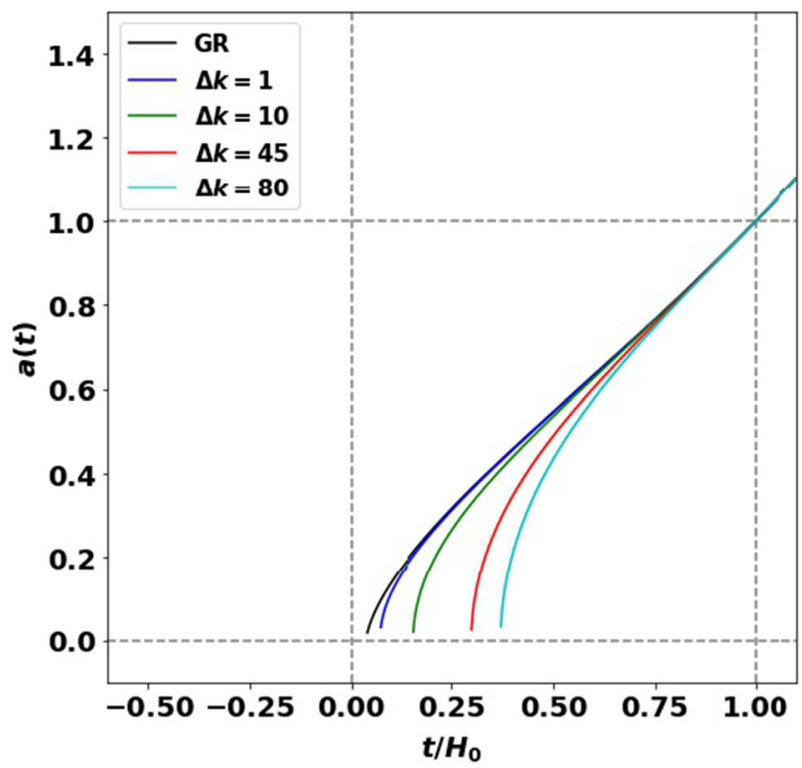

Fig. 14 Lin-lin plot of the scale parameter as a function of the universal time indicating a singular Big Bang and a finite age of the universe. GR refers to Einstein-Friedman cosmology

initial explosion is again supernova-like, but unlike the dynamics in the parameter Region II, no secondary inflation phase arises. This scenario is thus a copy of the conventional EinsteinFriedman cosmology but its dynamics including the onset of the dark energy era is shifted to smaller redshifts - presumably at odds with observations. 


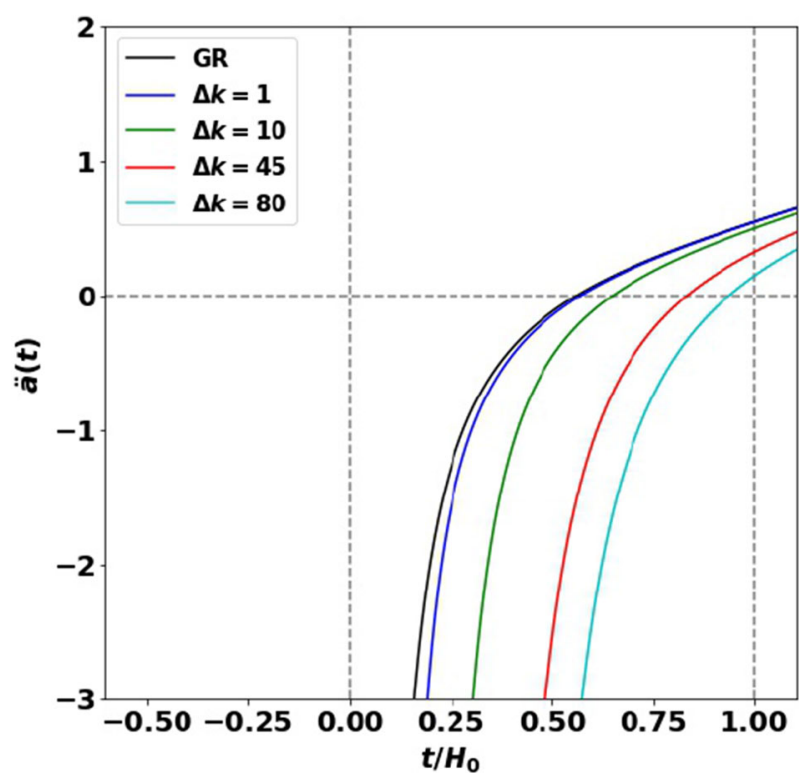

Fig. 15 Expansion acceleration $\ddot{a}(t)$ (units $H_{0}^{2}$ ) commences with a large negative value at the initial time $t_{i}$ but the deceleration decreases with increasing universal time

The general behavior of the dark energy and curvature functions, $f\left(a ; K_{0}\right)$ and $k\left(a ; K_{0}\right)$, for positive $K_{0}$ (and positive $g_{2}$ ) is discussed in "Appendix C.2." The energy conditions NEC, WEC and DEC are satisfied.

\subsection{Comparison with the SNeIa Hubble diagram}

We finally compare the CCGG cosmology model and the standard GR $\Lambda$ CDM model with the observational data via the relation between the distance modulus $\mu$ and the redshift $z$. The observational supernovae data come from Ref. [36]. Distance estimates from SNeIa light curves are derived from the luminosity distance

$$
d_{L}=\sqrt{\frac{L_{\mathrm{int}}}{4 \pi \mathcal{F}}}=a\left(t_{0}\right)(1+z) \int_{t_{0}}^{t} \frac{\mathrm{d} t^{\prime}}{a\left(t^{\prime}\right)}=(1+z) \int_{0}^{z} \frac{\mathrm{d} z^{\prime}}{H\left(z^{\prime}\right)},
$$

where $L_{\text {int }}$ is the intrinsic luminosity and $\mathcal{F}$ is the observed flux of the supernovae. Inserting the Hubble function for the CCGG model, Eq. (34), into Eq. (45) yields

$$
\begin{aligned}
& d_{L}=(1+z) \int_{0}^{z} d z^{\prime} \times \\
& {\left[C_{m}\left(1+z^{\prime}\right)^{3}-K_{0}\left(1+z^{\prime}\right)^{2}+C_{\Lambda} f\left(z^{\prime}\right)+\frac{\left(C_{\Lambda} f\left(z^{\prime}\right)+\frac{1}{4} C_{m}\left(1+z^{\prime}\right)^{3}\right)\left(\frac{3}{4} C_{m}\left(1+z^{\prime}\right)^{3}\right)}{\frac{1}{2} g_{2}-\frac{1}{4} C_{m}\left(1+z^{\prime}\right)^{3}-C_{\Lambda} f\left(z^{\prime}\right)}\right]^{-1 / 2} .}
\end{aligned}
$$

The logarithm of the luminosity distance is related to the flux (apparent magnitude, $m$ ) and luminosity (absolute magnitude, $M$ ) of the observed supernovae via the formula for the extinction-corrected distance modulus, $\mu=m-M=5 \log \frac{d_{L}}{M p c}+25$. The dependence of 

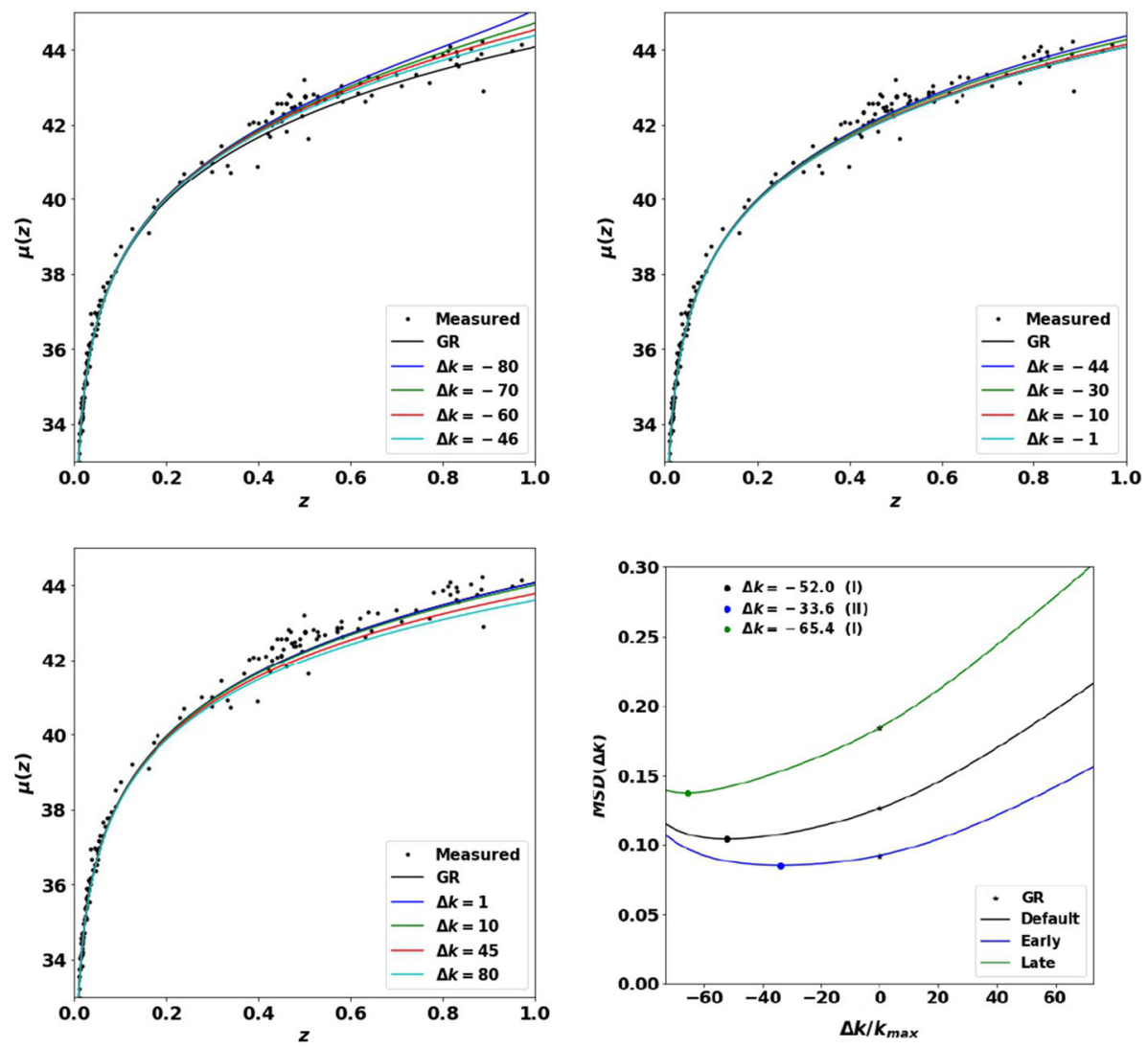

Fig. 16 The SNeIa Hubble diagrams are compared with the model prediction for the Regions I (top left), II (top right), III (bottom left). The plot at the bottom-right is the mean-square deviation for various sets of the $\Lambda \mathrm{CDM}$ parameter sets

the predicted distance modulus $\mu$ on the redshift $z$ is plotted for the parameter Regions I, II and III in Fig. 16 and compared with the observational data of the SNeIa Hubble diagram [36].

Obviously, the CCGG calculations for negative $K_{0}$ in the vicinity of $\bar{K}_{0} / k_{\max }=45.011$, for which the coupling constant $g_{2}$ vanishes and $g_{1}$ is large, are the best fit of the data. The quadratic scale-invariant gravity model thus seems to be preferred over Einstein-Hilbert. The mean-square deviation is minimized for $K_{0} / k_{\max }=-52$, i.e., in Region I, pointing at a nonsingular universe with steady inflation. Yet a sensitivity analysis (bottom right plot) reveals that slight modifications of the concordance parameter set (cf. Table 2) shift the optimum to Region II: For the "Late" datasets, we find the MSD minimum at $K_{0} / k_{\max }=-65.4$, but at $K_{0} / k_{\max }=-33.6$ for the "Early" dataset (CMB). Interestingly, the Early dataset yields a better fit with the observations of the late epoch ${ }^{22}$. Hence, the singular Big Bang with a secondary inflation phase is also a good scenario consistent with the Concordance Model, even more as it also satisfies the standard energy conditions (Table 2).

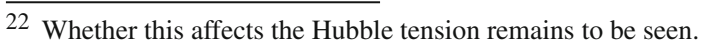


Table 2 The $\Lambda$ CDM parameter sets used for the sensitivity check of the Hubble diagram fit. The data are taken from Refs. [32] (= Default, applied throughout this paper), [19] (Late) and [33] (Early). The constants used in the equations are given by $C_{i}=\Omega_{i} h_{0}^{2} H_{100}^{2}$

\begin{tabular}{lllll}
\hline Data & $\Omega_{\Lambda}$ & $\Omega_{m}$ & $\Omega_{r}$ & $h_{0}$ \\
\hline Default & 0.69990 & 0.30000 & 0.00005 & 0.70903 \\
Late & 0.70000 & 0.30000 & 0.00005 & 0.74500 \\
Early & 0.68500 & 0.31500 & 0.00005 & 0.67400 \\
\hline
\end{tabular}

\section{The cosmological constant problem}

According to Eqs. (15) and (31), the observable cosmological constant is composed of three independent terms: the vacuum energy $g_{3} / M_{p}^{2}$, the "(A)dS curvature" $3 g_{2}$ and the presentday value of the Cartan contortion density, $P(1)$. This variety of contributions facilitates sufficient freedom to align the theoretical and observational values of the cosmological constant and thus provides a new perspective for resolving the so-called Cosmological Constant Problem $^{23}$. Any present-day vacuum energy density $g_{3}$ can, by a "suitable" choice of the necessarily nonvanishing (A)dS coupling constant $g_{2}$ and the Cartan contortion density, be made compatible with the present-day value of the cosmological constant.

To be more specific, provided $g_{3} \sim M_{p}^{4}>0$ and the unknown field $P(x)$ is neglected, the vacuum energy will be compensated to a value close to zero if the deformation parameter is negative of the order $g_{1} \approx-3 / 2$ [49], or $g_{2} / H_{0}^{2} \approx-10^{120}$. Such a large negative value is achievable with a very small negative value of the FLRW curvature parameter, $K_{0} \rightarrow 0_{-}$. Taking though the best fit to the Hubble diagram as discussed in Sect. 5.4, the curvature parameter $K_{0} / k_{\max }$ is found in the vicinity of $g_{2} \approx 0$. Then, the contribution of the Cartan contortion density cannot be neglected in the balance equation (31), giving the present-day scalar contortion density of the order $\frac{1}{4} P(1) \sim-M_{p}^{2}$.

However, assuming $g_{3}=0$, i.e., abandoning the "naked" cosmological constant that Einstein called "the biggest blunder of my life," gives for the best-fit parameters $K_{0} / k_{\max }=$ $-52.0,-65.4,-33.6$ the respective values $\frac{1}{3} \lambda_{0} / H_{0}^{2}=g_{2} / H_{0}^{2}=0.21,0.48,-0.62$. This is indeed at the order of magnitude of $\Omega_{\Lambda} \approx 0.7$, a remarkable fact especially in view of the uncertainty of the $\Lambda \mathrm{CDM}$ priors and the unknown value of the present-day contortion density. $g_{2}<0$, pointing to Region II, is applicable only if $g_{3} \leq 0$.

\section{Summary and conclusions}

The CCGG theory facilitates, in a mathematically rigorous way, a consistent description of the dynamics of space-time and matter. Applying the de Donder-Hamilton-Palatini framework of covariant canonical transformations, it unambiguously fixes the coupling of space-time to matter fields [44] and requires a quadratic momentum tensor term [5] extending the EinsteinHilbert theory. The canonical field equations are obtained by variation of the action integral with respect to the independent fields affine connection, metric and their conjugate momentum fields. Combining these field equations gives the Einstein equation of General Relativity extended by a quadratic Riemann-Cartan concomitant. Since the Schwarzschild and Kerr metrics are solutions of that extended (so-called CCGG) equation, all standard solar tests can be reproduced [44].

23 Similar conclusions with respect to the cosmological constant have been discussed elsewhere, see, for example, $[17,20]$. However, a thorough cosmological model is missing in that framework. 
Space-time in the CCGG theory is a dynamical medium endowed with kinetic energy and inertia. The strength of that inertia is determined by the dimensionless (deformation) parameter $g_{1}$ that must be finite in order to ensure the theory's intrinsic consistency. The dynamics of matter and space-time are intertwined such that only the total energy momentum, i.e., sum of stress and strain energies, is covariantly conserved. By requiring the stress-energy tensor to satisfy the covariant conservation law for any given metric, the resulting covariant conservation of the strain-energy tensor is in general possible only with an asymmetric affine connection. That leads to correction terms in the CCGG equation based on the then necessary presence of torsion of space-time. In the Friedman universe that torsion-dependent portion of the Ricci curvature scalar (called here Cartan contortion density) emerges as a new dynamical energy reservoir, expressed as a running cosmological constant that we call dark energy field. This and further curvature corrections invoked by space-time's inertia modify the cosmological dynamics.

The curvature constant of the FLRW metric, $K_{0}$, emerges as a new, and the only, free parameter of the theory and determines the deformation parameter $g_{1}$ and the deceleration parameter $q_{0}$, thus exposing $g_{1}$ to direct observations ${ }^{24}$.

Our numerical analysis presented here uses for comparative reasons the $\Lambda \mathrm{CDM}$ parameter set of the Concordance Model to identify three scenarios for the early evolution of the universe:

Scenario I emerges for negative $K_{0}$ and positive $g_{2}$ (and $g_{1}$ ). It describes a universe that starts off from a nonsingular bounce event into a steady inflation phase that gracefully exits into the current dark energy era. An interesting feature of this scenario is, in view of the so-called Hubble tension [37], the increasing Hubble function.

Scenario II-for negative $K_{0}$ and negative $g_{2}$-is a hard singular Big Bang initially similar to Einstein-Friedman cosmology but followed by a secondary inflation that, after a deceleration period, finally exits into the late dark energy era. Both scenarios are consistent with the late epoch SNeIa Hubble diagram.

This is not the case for Scenario III with $K_{0}$ and $g_{2}$ both positive. Here, the universe undergoes an evolution that is very similar to the standard Einstein-Friedman cosmology but with the Big Bang shifting to later times with increasing $K_{0}$.

While for the total effective EOS $\omega \geq-1$ holds in Regions II and III, it is not the case in Region I. Hence, NEC, WEC and DEC are satisfied in Regions II and III but violated in Region I.

We conclude that the quadratic Riemann-Cartan invariant introduced by the CCGG formalism is a necessary extension of the Einstein-Hilbert theory facilitating a viable contribution to cosmology ${ }^{25}$. The model is, unlike other modified gravity models, formally derived from first principles. The dark energy and inflation emerge as effects of the extended geometry, with no matter fields beyond the standard model required. And new light is shed on the cosmological constant problem, and perhaps even on the Hubble tension.

The initial analysis presented here is compatible with the $\Lambda \mathrm{CDM}$ parameter set, but provides due to its additional parameter, more flexibility to reproduce observations. A particular example is the decoupling of the FLRW and apparent curvatures, and the correction of the deceleration parameter. In order to substantiate the above conjectures, a comprehensive comparison of the theory with the full body of observational evidence including early-universe

\footnotetext{
24 For clarity: the deformation parameters $g_{1} \sim 1 / g_{2}$ and $K_{0}$ are equivalent via Eq. (38) reducing the number of new independent parameters to just one.

25 The nonsingular solutions of CCGG cosmology have also been investigated in [7]. Even if torsion is neglected, the CCGG version of the Dirac equation gives rise to a curvature-dependent mass correction that drives inflation [6].
} 
data is needed. The impact of contortion-related tensor terms and that of spin-carrying matter are further challenging areas for future investigations.

Acknowledgements Open Access funding provided by Projekt DEAL. This work has been supported by the Walter Greiner Gesellschaft zur Förderung der physikalischen Grundlagenforschung e.V. DV and JK especially thank the Fueck-Stiftung for support.

The authors also wish to thank Dirk Kehm for helping with the Hubble diagram analysis and Horst Stöcker, Andreas Redelbach, Eduardo Guendelman, David Benisty, Patrick Liebrich, Julia Lienert, Peter Hess and Matthias Hanauske for stimulating discussions.

Data Availability Statement This manuscript has associated data in a data repository. [Authors' comment: All data generated in this are reproducible using the formulas listed in the document. External, experimental data, are from the publications quoted in the list of references.]

Open Access This article is licensed under a Creative Commons Attribution 4.0 International License, which permits use, sharing, adaptation, distribution and reproduction in any medium or format, as long as you give appropriate credit to the original author(s) and the source, provide a link to the Creative Commons licence, and indicate if changes were made. The images or other third party material in this article are included in the article's Creative Commons licence, unless indicated otherwise in a credit line to the material. If material is not included in the article's Creative Commons licence and your intended use is not permitted by statutory regulation or exceeds the permitted use, you will need to obtain permission directly from the copyright holder. To view a copy of this licence, visit http://creativecommons.org/licenses/by/4.0/.

\section{Appendices}

\section{A Derivation of the Friedman equations}

The material content of the Friedman model universe is perfect fluids made of classical particles and radiation. The stress-energy tensor for a perfect fluid with the density $\rho$ and pressure $p$ is symmetric. In the comoving frame,

$$
T_{v}^{\mu}=\sum_{i=r, m} \operatorname{diag}\left(\rho_{i},-p_{i},-p_{i},-p_{i}\right)
$$

$\rho_{i}$ and $p_{i}$ are functions of the global time $t$ only, and the index $i$ tallies just two basic types of matter, namely particles ("dust," $i=m$ ) and radiation $(i=r)$. Of course, the particle matter is itself a sum over all standard-model particles. Radiation, on the other hand, includes not only genuine photon energy density but also contribution from highly relativistic particles where mass is negligible compared to their kinetic energy, i.e., neutrinos.

The equation of state (EOS) for a perfect fluid is assumed [51] to have the generic form

$$
p_{i}=\omega_{i} \rho_{i}
$$

where $\omega_{m}=0$ and $\omega_{r}=\frac{1}{3}$. The requirement that the covariant divergence of the energymomentum tensor of this matter content vanishes implies the scaling law [2,51]:

$$
\rho_{i}(t) \sim a^{-n_{i}} \quad \Rightarrow \quad \rho_{i} a^{n_{i}}=\mathrm{const},
$$

with the definition $n_{i} \equiv 3\left(\omega_{i}+1\right)$, i.e., $n_{r}=4$ and $n_{m}=3$.

We wish now to solve the CCGG-Friedman Eq. (29), simplified by setting $P^{\prime(\mu \nu)}=0$ and $\xi^{\mu \nu}=0$, for classical, spinless matter forming perfect fluids:

$$
g_{1} \bar{Q}^{\mu \nu}-\frac{1}{8 \pi G}\left[\bar{G}^{\mu \nu}-g^{\mu \nu} \Lambda(x)\right]=\bar{T}^{\mu \nu} .
$$


The components of the Riemann tensor are derived from the FLRW metric (32) using the Levi-Civita relation. The component $\mu=v=0$ of Eq. (A.4) yields:

$$
-8 \pi G g_{1}\left[\left(\frac{\dot{a}^{2}+K_{0}}{a}\right)^{2}-\ddot{a}^{2}\right]+\dot{a}^{2}+K_{0}-\frac{1}{3} \Lambda_{0} f(a) a^{2}=\frac{8 \pi G}{3} a^{2} \sum_{i=r, m} \rho_{i} .
$$

Dot denotes the derivative with respect to the universal time $t$. For the $\mu=v=1$ component, we obtain in addition

$$
8 \pi G g_{1}\left[\left(\frac{\dot{a}^{2}+K_{0}}{a}\right)^{2}-\ddot{a}^{2}\right]+2 a \ddot{a}+\dot{a}^{2}+K_{0}-\Lambda_{0} f(a) a^{2}=-8 \pi G a^{2} \sum_{i=r, m} p_{i},
$$

with $f(a)$ defined in Eq. (33). For the trace of Eq. (A.4), which is independent of the presence of the traceless quadratic Riemann term, we obtain

$$
a \ddot{a}+\dot{a}^{2}-2 M a^{2}+K_{0}=0
$$

where

$$
M:=\frac{1}{3}\left[2 \pi G \sum_{i}\left(\rho_{i}-3 p_{i}\right)+\Lambda_{0} f(a)\right]=\frac{1}{3}\left[2 \pi G \sum_{i}\left(\rho_{i}\left(4-n_{i}\right)+\Lambda_{0} f(a)\right] .\right.
$$

Other combinations of the indices $\mu$ and $v$ either vanish or reproduce the above equations. Moreover, adding Eq. (A.5) and Eq. (A.6) yields Eq. (A.7), so only two of the three equations need to be considered.

To touch base with standard Friedman cosmology, we first show that with the quadratic Riemann term omitted (i.e., setting here formally $g_{1}=0$ and $f(a) \equiv 1$, and keeping $\lambda=\Lambda_{0}$ constant), we recover the conventional Friedman equations. Calculating the difference of Equations (A.5)-(A.6) yields

$$
a \Lambda_{0}-3 \ddot{a}=4 \pi G a \sum_{i}\left(\rho_{i}+3 p_{i}\right)
$$

which can be resolved for $\ddot{a}$ :

$$
\frac{\ddot{a}}{a}=-\frac{4 \pi G}{3} \sum_{i}\left(\rho_{i}+3 p_{i}\right)+\frac{1}{3} \Lambda_{0} .
$$

In a similar way, we obtain from Eq. (A.7) with the definition of the Hubble function $H:=$ $\dot{a} / a$ :

$$
\frac{\ddot{a}}{a}+H^{2}+\frac{K_{0}}{a^{2}}=\frac{2}{3}\left[2 \pi G \sum_{i}\left(\rho_{i}-3 p_{i}\right)+\Lambda_{0}\right] .
$$

Inserting Eq. (A.10) yields the well-known Friedman equation based on General Relativity:

$$
H^{2}(a)=\frac{8 \pi G}{3} \sum_{i} \rho_{i}-\frac{K_{0}}{a^{2}}+\frac{1}{3} \Lambda_{0} .
$$

It is rather straightforward to rerun the derivation 
with $g_{1} \neq 0$ and look for modifications arising from the quadratic term in the CCGG equation. Inserting Eq. (A.7) in Eq. (A.5) yields, after multiplication with $a^{-2}$, the modified Friedman equation

$$
\begin{aligned}
H^{2}(a)= & -\frac{K_{0}}{a^{2}}+\frac{1}{3} \Lambda(a)+ \\
& +\left[\frac{8 \pi G}{3} \sum_{i} \rho_{i}-\frac{(8 \pi G)^{2}}{3} g_{1} M(a) \sum_{i}\left(\rho_{i}-3 p_{i}\right)\right]\left[1-32 \pi G g_{1} M(a)\right]^{-1} .
\end{aligned}
$$

With the equation of state (A.2) and the scaling properties (A.3), this equation reads:

$$
H^{2}(a)=\frac{8 \pi G}{3} \sum_{i} \rho_{i}-\frac{K_{0}}{a^{2}}+\frac{1}{3} \Lambda(a)+g_{1} \frac{8 \pi G}{3} \sum_{i} \rho_{i} n_{i} \frac{8 \pi G M(a)}{\left(1-32 \pi G g_{1} M(a)\right)} .
$$

Obviously, while the first three terms on the r.h.s. formally correspond to the conventional Friedman Eq. (A.10), the last term is generated by the quadratic term in Eq. (9) and vanishes with $g_{1}=0$.

It is convenient to define the constants

$$
C_{i}:=\frac{8 \pi G}{3} \rho_{i} a^{n_{i}}=\text { const. }, \quad i=r, m .
$$

and

$$
\begin{array}{ll}
C_{K}:=-K_{0} & \omega_{K}=-\frac{1}{3}, n_{K}=2 \\
C_{\Lambda}:=\frac{1}{3} \Lambda_{0} & \omega_{\Lambda}=-1, n_{\Lambda}=0 .
\end{array}
$$

The modified Friedman equation (A.13) then becomes

$$
H^{2}(a)=\sum_{i=r, m, K} C_{i} a^{-n_{i}}+C_{\Lambda} f(a)+g_{1} \frac{32 \pi G M(a)}{\left(1-32 \pi G g_{1} M(a)\right)} \sum_{i=r, m} \frac{1}{4} C_{i} n_{i} a^{-n_{i}} .
$$

with $M(a)$, in Eq. (A.8), rewritten as

$$
M(a)=\frac{1}{4} C_{m} a^{-3}+C_{\Lambda} f(a) .
$$

The present-day energy densities of matter including dark matter, of radiation including photons and neutrinos, are expressed in the text by the cosmological constant $C_{i}=\Omega_{i} H_{0}^{2}$. Where not stated otherwise, we use the "Default" values $\Omega_{i}$ and $H_{0}$ listed in Table 2 ("Concordance Model" or " $\Lambda$ Cold Dark Matter," $\Lambda$ CDM model of standard cosmology, see, for example, $[8,32])$, and

$$
\begin{aligned}
M_{p} & =\frac{1}{\sqrt{8 \pi G}} \approx 2.428 \times 10^{18} \mathrm{GeV} \\
\left|k_{\mathrm{obs}}\right| & \leq k_{\max }:=0.005 \times H_{0}^{2} .
\end{aligned}
$$

With the initial conditions, $f(1)=1$ and $k(1)=0$, the value $H_{0} \equiv H(a=1)$ of the Hubble constant is reproduced from Eq. (A.16):

$$
\begin{aligned}
H_{0} & \approx 1.499 \times 10^{-42} \mathrm{GeV}=70.259 \frac{\mathrm{km}}{\mathrm{s} \mathrm{Mpc}}=h_{0} H_{100}=0.70259 H_{100} \\
H_{100} & :=100 \frac{\mathrm{km}}{\mathrm{s} \mathrm{Mpc}}=2.135 \times 10^{-42} \mathrm{GeV} .
\end{aligned}
$$




\section{B Derivation of the ODE for $f(a)$}

In this section, we pursue the above analysis of the CCGG-Friedman model, in Eq. (A.16) or Eq. (34), to derive the equation of motion for the dark energy function $C_{\Lambda} f(a)$.

It is helpful at this point to set

$$
-V(a):=a^{2} H^{2}(a)+K_{0}
$$

and rewrite Eq. (34) in the form of the ordinary, one-dimensional differential equation

$$
\dot{a}^{2}+V(a)=-K_{0} .
$$

This equation describes the radial expansion dynamics of the universe. Formally, though, also the conservation of the "total energy," $-K_{0}$, of a fictitious point particle with "kinetic energy" $\dot{a}^{2}$, mass 2 and "potential energy"

$$
V(a)=-C_{m} a^{-1}-C_{r} a^{-2}-C_{\Lambda} a^{2} f(a)-\frac{M(a)}{\frac{1}{2} g_{2}-M(a)}\left(\frac{3}{4} C_{m} a^{-1}+C_{r} a^{-2}\right) .
$$

Obviously, any radial motion comes to a hold once $\dot{a}=0$. Hence,

$$
V(a)>-K_{0}
$$

defines a turning point for the expansion (or contraction) of the universe as the expansion velocity, $\dot{a}=\sqrt{-V(a)-K_{0}}$, vanishes or even becomes imaginary there. For $\ddot{a}>0$, i.e. if

$$
\frac{V(a)}{a}+2 M a>0,
$$

the radial expansion accelerates. The time period in which this is the case is called inflation.

Differentiating now Eq. (B.2) with respect to the universal time $t$ yields

$$
\ddot{a}=-\frac{1}{2} \frac{\mathrm{d} V(a)}{d a} .
$$

On the other hand, combining Eqs. (A.7) and (B.1) gives

$$
\ddot{a}=\frac{V(a)}{a}+2 M a \text {. }
$$

Comparing expressions (B.6) and (B.7) for $\ddot{a}$, we conclude that the identity

$$
\frac{\mathrm{d} V(a)}{\mathrm{d} a}+\frac{2}{a} V(a)=4 M a
$$

must hold. Splitting (B.3),

$$
V\left(a ; f ; g_{1}\right)=V_{0}(a ; f)+V_{c}(a),
$$

separates the Einstein-Friedman portion,

$$
V_{0}(a ; f):=-C_{m} a^{-1}-C_{r} a^{-2}-C_{\Lambda} a^{2} f(a),
$$

from the "curvature correction term,"

$$
V_{c}(a):=-\frac{M(a ; f)}{\frac{1}{2} g_{2}-M(a ; f)}\left(\frac{3}{4} C_{m} a^{-1}+C_{r} a^{-2}\right),
$$


that depends on $g_{2}$ and $f(a)$ and vanishes for $g_{1}=0$, i.e. for $g_{2}=\infty$. The identity Eq. (B.8) applied to $V_{0}$ gives

$$
\frac{\mathrm{d} V_{0}(a ; f)}{\mathrm{d} a}+\frac{2}{a} V_{0}(a ; f)=-4 a M(a ; f)-C_{\Lambda} f^{\prime}(a) a^{2},
$$

where $f^{\prime}=\mathrm{d} f / \mathrm{d} a$. Obviously, Eq. (B.8) holds for $g_{1}=0$ and $f(a)=$ const., i.e., for the conventional Einstein-Friedman scale potential:

$$
\frac{\mathrm{d} V_{0}(a ; 1)}{\mathrm{d} a}+\frac{2}{a} V_{0}(a ; 1)+4 a M(a ; 1) \equiv 0 .
$$

A constant cosmological constant violates the CCGG equations, though, as for the "curvature correction" term (and $f(a)=$ const,$C_{m} \neq 0$ ) we get

$$
\frac{\mathrm{d} V_{c}(a)}{\mathrm{d} a}+\frac{2}{a} V_{c}(a)+4 M a \neq 0 .
$$

For a nonconstant $f(a)$, a straightforward calculation gives

$$
\begin{aligned}
\frac{\mathrm{d} V_{c}(a)}{\mathrm{d} a}+\frac{2}{a} V_{c}(a)= & -\frac{\frac{3}{4} C_{m} a^{-2} M}{\frac{1}{2} g_{2}-M} \\
& +\frac{\frac{1}{2} g_{2}}{\left(\frac{1}{2} g_{2}-M\right)^{2}} \frac{3}{4} C_{m} a^{-4}\left(\frac{3}{4} C_{m} a^{-1}+C_{r} a^{-2}\right) \\
& -C_{\Lambda} f^{\prime}(a) \frac{\frac{1}{2} g_{2}}{\left(\frac{1}{2} g_{2}-M\right)^{2}}\left(\frac{3}{4} C_{m} a^{-1}+C_{r} a^{-2}\right) .
\end{aligned}
$$

Inserting Eq. (B.12) and Eq. (B.13) into the identity (B.8) gives, after some algebra,

$$
\begin{aligned}
& \frac{\mathrm{d} f}{\mathrm{~d} a}=\frac{3 C_{m}}{4 C_{\Lambda}} a^{-4} \times \\
& \quad \frac{\frac{1}{2} g_{2}\left(\frac{3}{4} C_{m} a^{-3}+C_{r} a^{-4}\right)-\left(\frac{1}{2} g_{2}-\frac{1}{4} C_{m} a^{-3}-C_{\Lambda} f\right)\left(\frac{1}{4} C_{m} a^{-3}+C_{\Lambda} f\right)}{\frac{1}{2} g_{2}\left(\frac{3}{4} C_{m} a^{-3}+C_{r} a^{-4}\right)+\left(\frac{1}{2} g_{2}-\frac{1}{4} C_{m} a^{-3}-C_{\Lambda} f\right)^{2}} .
\end{aligned}
$$

This is an ordinary first-order differential equation (ODE) that has a unique solution for a given initial condition, here $f(1)=1$. Notice that this ODE is not changed if we replace the constants $C_{i}$ by $\Omega_{i}$, and $g_{2}$ by $g_{2} / H_{0}^{2}$.

\section{Calculated dark energy and curvature corrections and the resulting EOS}

C.1 $K_{0}<0$

The normalized effective dark energy function $f\left(a ; K_{0}\right)$ modifies the Hubble function relative to the Einstein-Friedman theory via the correction term $\Omega_{\Lambda}\left(f\left(a ; K_{0}\right)-1\right)$ plotted in the first row in Fig. 17. It is negative in the early epoch of the universe ${ }^{26}$. Asymptotically, though, it vanishes paving the way to the dark energy age with $f \sim 1$. This can be understood as an

26 In GR, a negative, time-independent cosmological constant in the early universe and cyclic inflation scenarios have been discussed in $[9,10]$. 

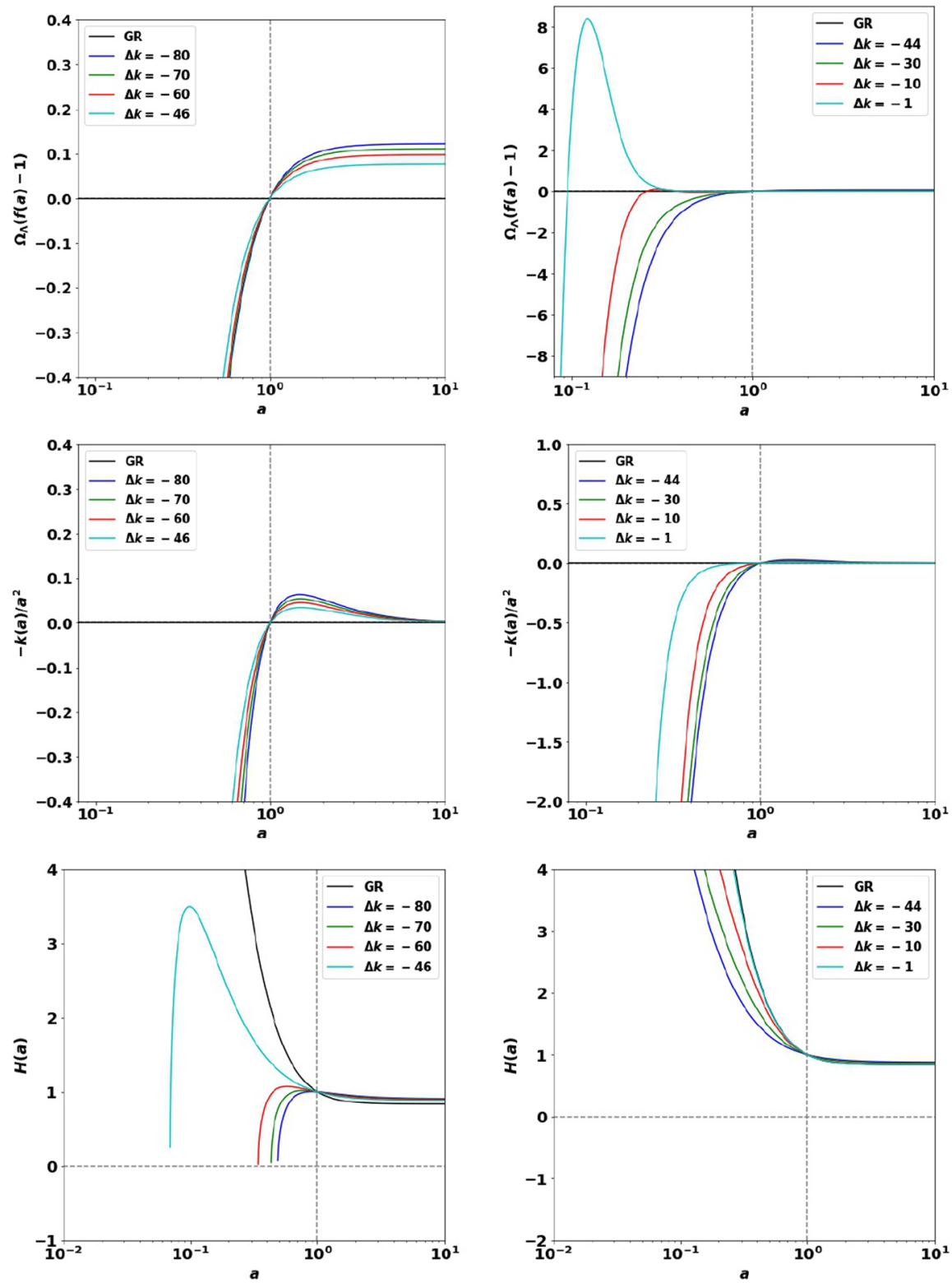

Fig. 17 Scale dependence of the dark energy term (top row), and the curvature term (middle row) facilitating the geometrical corrections to the Hubble function, and the Hubble function $H(a)$ (bottom row) in parameter Regions I (left column) and II (right column). The units used are $1, H_{0}^{2}$, and $H_{0}$, respectively

effect of the Cartan contortion density that initially appears to be high and acts as a storage of potential energy. That energy is gradually released to drive the inflation dynamics ${ }^{27}$.

27 Space-time can be thought of as a knitted elastic medium that can be stretched to some maximum but then rebounds to its equilibrium size. Internally, we call our model a universe "contorted space-time." 

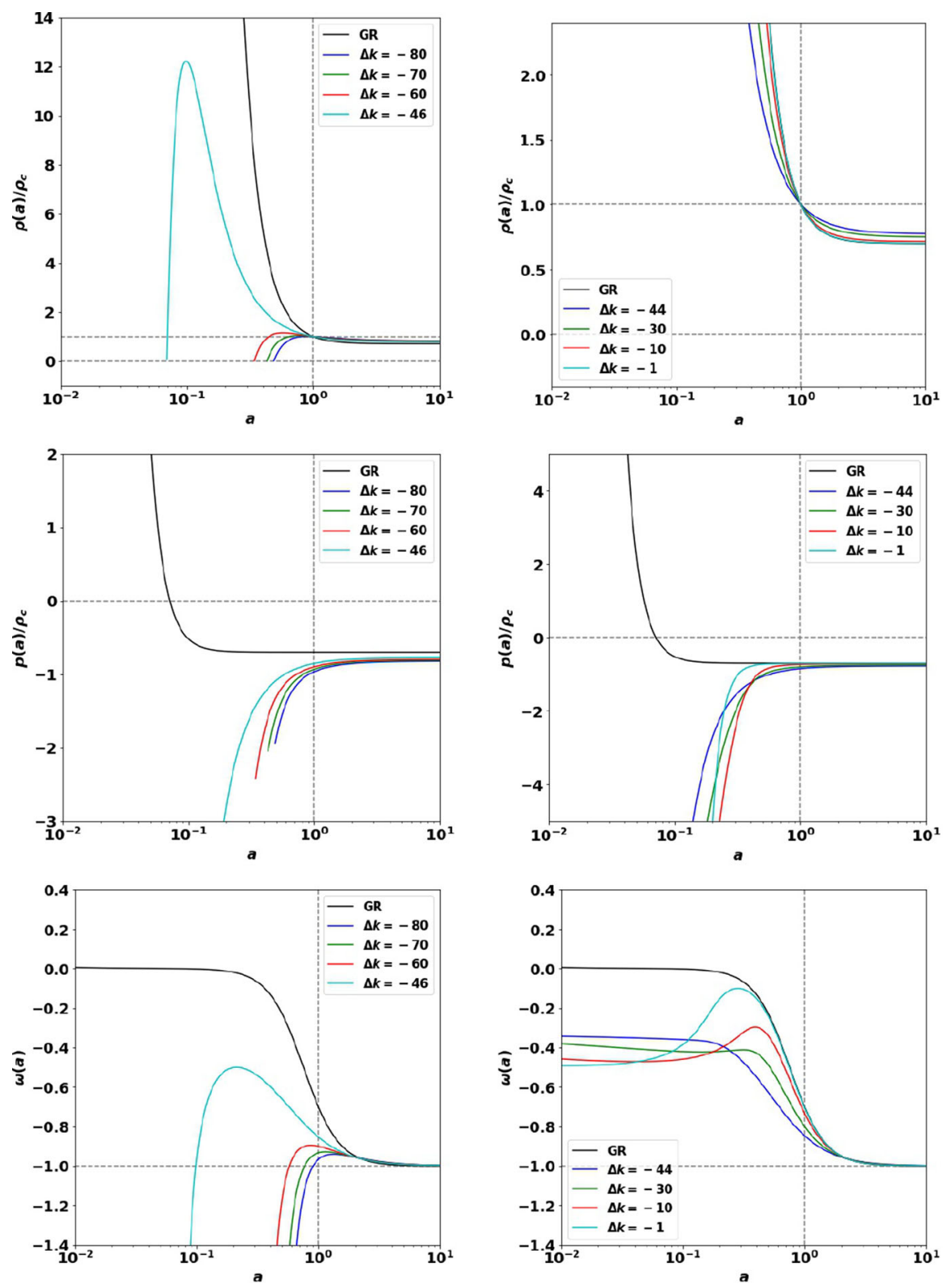

Fig. 18 Scale dependence of the energy density (top row) and pressure (middle row) of the total content of the universum, i.e., (dark) matter, radiation and geometrical corrections, relative to the critical density $\rho_{\text {crit }}$. The bottom row displays the resulting equation of state. Left column: parameter Region I, right column: parameter Region II. The black lines depict the Einstein-Friedman cosmology. The GR-EOS undergoes a transition from a matter to a dark energy dominated universe

In Region I, with $g_{2}>0$, the present dark energy reservoir is almost depleted. $f(a)$ reaches its maximum for $a>1$. In Region II, on the other hand, the dark energy function overshoots the asymptotic value of 1 to large positive values. Thus, in this case, after complete 

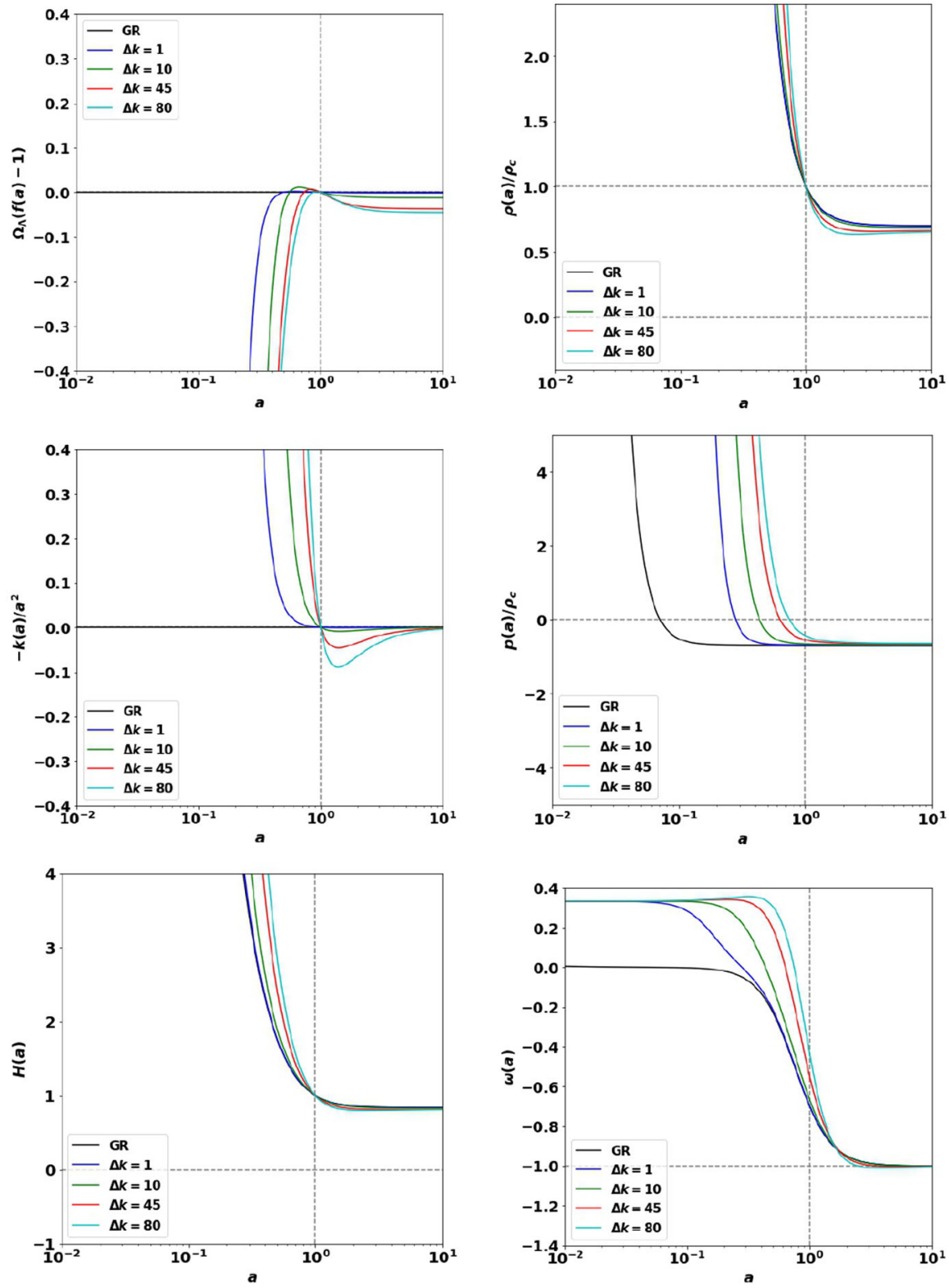

Fig. 19 Parameter Region III. Left column from top to bottom: Geometric corrections of the Hubble function relative to Einstein-Friedman cosmology from the dark energy and curvature functions, and the resulting Hubble function, $H(a)$. The units in the plots are $1, H_{0}^{2}$ and $H_{0}$, respectively. Right column: The relative total energy density and pressure, and the resulting equation of state $\omega(a) \geq-1$

depletion, the contortion density restarts absorbing energy again decelerating the expansion until it reaches a saturation value, but with a slightly modified cosmological constant. The overshoot disappears though for $g_{2} \rightarrow-\infty$. 
The effective curvature is, independently of the sign of $g_{2}$, initially positive, dropping steeply to the present-day value (here $k(1)=k_{\text {obs }}=0$ ) to asymptotically arrive at the constant value $K_{0}$. The plot in the middle row of Fig. 17 displays the curvature correction term $-k(a) /\left(a H_{0}\right)^{2}$ that directly adds to the formula for standard Hubble function $H^{2}(a)$ and so reinforces the dark energy correction and supports the acceleration of the scale expansion. The corresponding Hubble function is plotted in the bottom row of Fig. 17. The impact of the above correction terms is clearly visible.

In Region I, the Hubble function $H(a)$ increases from the edge of the forbidden area at $a=a_{i}$ and passes through a maximum in the recent past ${ }^{28}$ to approach a constant value $H(a) \approx H_{0}$ for $a>1$. For $a \ll 1, H(a)$ descends as the correction terms approach $-\infty$ as $a^{-n}$ with the effective power of $n>4$ and thus dominate the radiation term of the Einstein-Friedman cosmology. The peak is obviously the result of a slight mismatch in the $a$-dependence of the dark energy and curvature corrections. In Region II, the corrections terms diverge with $n \lesssim 4$ and are just a minor correction to the Hubble function.

The total energy density of the universe, encompassing matter, radiation and dynamical space-time geometry is always positive, see the first row in Fig. 18. The pressure, see the middle row in Fig. 18, is always negative in alignment with ongoing expansion. In Region I, the total effective EOS (bottom row) approaches a negative value $\omega\left(a_{i}\right) \ll-1$ at the bounce scale, which proves the violation of the standard energy conditions.

C.2 $K_{0}>0$

In Region III, the dark energy function and the curvature correction depend on the scale parameter in a similar way, and their contribution to the Hubble function almost cancels out (see the left column of Fig. 19), resulting in an overall evolution of the universe that is rather similar to the flat Einstein-Friedman cosmology. The latter is the limiting case $K_{0} \rightarrow \infty$. That similarity is also obvious from the plots of the total energy density, pressure and EOS (right column). NEC, WEC and DEC but not SEC are satisfied.

\section{References}

1. R. Aldrovandi, J.G. Pereira, De Sitter relativity: a new road to quantum gravity? Found. Phys. 39, 1-19 (2009)

2. E.E. Arsznov G.B. Thompson, D.D. Reid, D.W. Kittell, The picture of our universe: a view from modern cosmology (2002) arXiv:astro-ph/0209504

3. S. Basilakos, N.E. Mavromatos, J. Sola, Gravitational and chiral anomalies in the running vacuum universe and matter-antimatter asymmetry (2019), arXiv:1907.04890 [hep-ph]

4. D. Benisty, E.I. Guendelman, E.N. Saridakis, The scale factor potential approach to inflation (2019), (arXiv:1909.01982)

5. D. Benisty, E.I. Guendelman, D. Vasak, J. Struckmeier, H. Stoecker, Quadratic curvature theories formulated as covariant canonical gauge theories of gravity. Phys. Rev. D 98, 106021 (2018). (arXiv: $1809.10447 \mathrm{v} 1$ [gr-qc])

6. D. Benisty, E.I. Guendelman, E.N. Saridakis, H. Stoecker, J. Struckmeier, D. Vasak, Inflation from fermions with curvature-dependent mass. Phys. Rev. D 100, 043523 (2019). (arXiv:1905.03731 [gr-qc])

7. D. Benisty, D. Vasak, E. Guendelman, J. Struckmeier, Energy transfer from space-time into matter and a bouncing inflation from covariant canonical gauge theory of gravity. Mod. Phys. Lett. A 34(21), 1950164 (2019). (arXiv:1807.03557)

8. C. Bernal, V.H. Cardenas, V. Motta, Assymetry in the reconstructed deceleration parameter. Phys. Lett. B 765, 163-170 (2017)

28 Interestingly, an increasing Hubble constant is consistent with observational conclusions in Ref. [38]. 
9. T. Biswas, T. Koivisto, A. Mazumdar, Could our universe have begun with $-\lambda$ ? (2012), arXiv:1105.2636 [astro-ph.CO]

10. T. Biswas, A. Mazumdar, Inflation with a negative cosmological constant. Phys. Rev. D 80, 023519 (2009)

11. S.D. Brechet, M.P. Hobson, A.N. Lasenby, Weyssenhoff fluid dynamics in general relativity using a $1+3$ covariant approach. Class. Quant. Grav. 24, 6329 (2007)

12. C.G. Böhmer, P. Bronowski, The homogeneous and isotropic Weyssenhoff fluid. Ukr. J. Phys. 55, 607 (2010)

13. S. Capozzielio, F.S.N. Lobo, J.P. Mimoso, Energy conditions in modified gravity. Phys. Lett. B 730, 280-283 (2014)

14. S. Capozziello, Curvature quintessence. Int. J. Mod. Phys. D 11, 483 (2002)

15. S. Capozziello, S. Carloni, A. Troisi, Quintessence without scalar fields. Recent Res. Dev. Astron. Astrophys. 1, 625 (2003). (arXiv:astro-ph/0303041)

16. H. Chen, F.-H. Ho, J.M. Nester, C.-H. Wang, H.-J. Yo, Cosmological dynamics with propagating lorentz connection modes of spin zero. JCAP 0910, 027 (2009)

17. P. Chen, Gauge theory of gravity with de Sitter symmetry as a solution to the cosmological constant problem and the dark energy puzzle. Mod. Phys. Lett. A 25(33), 2795 (2010)

18. T. De Donder, Théorie Invariantive Du Calcul des Variations (Gaulthier-Villars \& Cie, Paris, 1930)

19. S. Dhawan, D. Brout, D. Scolnic, A. Goobar, A.G. Riess, V. Miranda, Cosmological model insensitivity of local $H_{0}$ from the Cepheid distance ladder (2020), arXiv: 2001.09260 astro-ph.CO

20. G.F.R. Ellis, The trace-free Einstein equations and inflation. Gen. Relativ. Gravit. 46, 1619 (2014)

21. L. Fabbri, P.D. Mannheim, Continuity of the torsionless limit as a selection rule for gravity theories with torsion. Phys. Rev. D 90, 024042 (2014)

22. A. Friedman, Über die Krümmung des Raumes. Z. Phys. 10, 377 (1922)

23. K. Hayashi, T. Shirafuji, Gravity from poincaré gauge theory of the fundamental particles I. Prog. Theor. Phys. 64(3), 866 (1980)

24. F.W. Hehl, P. von der Heyde, G.D. Kerlick, J.M. Nester, General relativity with spin and torsion: foundations and prospects. Rev. Mod. Phys. 48(3), 393 (1976)

25. F. Johansson, Version 1.1.0 (2018), http://mpmath.org

26. P. Jordan, Bemerkungen zur Kosmologie. Ann. Phys. 428(1), 64-70 (1939)

27. T.W.B. Kibble, Symmetry breaking in non-Abelian gauge theories. Phys. Rev. 155, 1554-1561 (1967)

28. P. Minkowski, On the cosmological equations in the presence of a spatially homogeneous torsion field. Phys. Lett. 173, 247 (1986)

29. R. Myrzakulov, S.D. Odintsov, L. Sebastiani, Inflationary universe from higher-derivative quantum gravity. Phys. Rev. D 91:8, 083529 (2015)

30. Y.N. Obukhov, V.A. Korotky, The Weyssenhoff fluid in Einstein-Cartan theory. Class. Quant. Gravity 4, 1633 (1987)

31. Y.N. Obukhov, R. Tresguerres, Hyperfluid - a model of classical matter with hypermomentum. Phys. Lett. A 184, 17-22 (1993)

32. Planck collaboration. Planck, results. XIII. Cosmological parameters. Astron. Astrophys. 594(A13), 2016 (2015)

33. Planck Collaboration. Planck 2018 results. VI. Cosmological parameters (2019), arXiv:1807.06209v2

34. N. Poplawski, The simplest origin of the Big Bounce and Inflation. Int. J. Mod. Phys. 27, 1847020 (2018). (arXiv:1801.08076)

35. J. Queiruga, Non-metric construction of spacetime defects (2019), arXiv:1912.10296

36. A.G. Riess, L.-G. Strolger, J. Tonry, S. Casertano, H.C. Ferguson, B. Mobasher, P. Challis, A.V. Filippenko, S. Jha, W. Li, R. Chornock, R.P. Kirshner, B. Leibundgut, M. Dickinson, M. Livio, M. Giavalisco, C.C. Steidel, N. Benitez, Z. Tsvetanov, Type-I supernova discoveries at $z>1$ from the hubble space telescope: evidence for past deceleration and constraints on dark energy evolution. Astrophys. J. 607, 665 (2004). (arXiv:astro-h/0402512)

37. A.G. Riess, The expansion of the Universe is faster than expected. Nat. Rev. Phys. 2, 10-11 (2019)

38. G. Risaliti, E. Lusso, Cosmological constraints from the Hubble diagram of quasars at high redshifts. Nat. Astron. 3, 272-277 (2019)

39. D.W. Sciama, in Recent Developments, in General Relativity, ed. by C. Misner, L. Witten, The analogy between charge and spin in general relativity, (Pergamon Press, Oxford, 1962), pp. 415-439

40. A.A. Starobinsky, A new type of isotropic cosmological models without singularity. Phys. Lett. B 91, 99 (1980)

41. A.A. Starobinsky, Dynamics of phase transition in the new inflationary universe scenario and generation of perturbations. Phys. Lett. B 117, 175 (1982)

42. J. Struckmeier, Generalized U(N) gauge transformations in the realm of the extended covariant Hamilton formalism of field theory. J. Phys. G Nucl. Phys. 40, 015007 (2013) 
43. J. Struckmeier, J. Muench, P. Liebrich, M. Hanauske, J. Kirsch, D. Vasak, L. Satarov, H. Stoecker, Canonical transformation path to gauge theories of gravity-II: space-time coupling of spin-0 and spin-1 particle fields. Int. J. Mod. Phys. E 28, 1950007 (2019). (arXiv:1711.10333 [gr-qc])

44. J. Struckmeier, J. Muench, D. Vasak, M. Hanauske, J. Kirsch, H. Stoecker, Canonical transformation approach to gauge theories of gravity I. Phys. Rev. D 95, 124048 (2017). (arXiv:1704.07246)

45. J. Struckmeier, A. Redelbach, Covariant Hamiltonian field theory. Int. J. Mod. Phys. E 17, 435-491 (2008)

46. J. Struckmeier, D. Vasak, H. Stoecker, Extended canonical field theory of matter and space-time. Astron. Nachr. 336(8/9), 731-738 (2015)

47. G. Unger, N. Poplawski, Big bounce and closed universe from spin and torsion. Astron. J. 870, 78 (2019)

48. R. Utiyama, Invariant theoretical interpretation of interaction. Phys. Rev. 101(5), 1597 (1956)

49. D. Vasak, J. Kirsch, D. Kehm, J. Struckmeier, Covariant canonical gauge gravitation and cosmology. J. Phys. Conf. Ser. 1194, 012108 (2019). (arXiv:1812.00578)

50. S. Weinberg, The cosmological constant problem. Rev. Mod. Phys. 61, 1 (1989)

51. S. Weinberg, Gravitation and Cosmology: Principles and Applications of the General Theory of Relativity (WSE, New York, 1972)

52. C. Wetterich, Cosmology and the fate of dilatation symmetry. Nucl. Phys. B 302, 668 (1988)

53. C. Wetterich, Inflation, quintessence, and the origin of mass. Nucl. Phys. B 897, 111 (2015) 